\title{
Quantitative comparison of electrically induced spin and orbital polarizations in heavy-metal/3d-metal bilayers
}

\author{
Leandro Salemi $\odot,{ }^{*}$ Marco Berritta, and Peter M. Oppeneer $\odot$ \\ Department of Physics and Astronomy, P.O. Box 516, Uppsala University, SE-75 20 Uppsala, Sweden
}

(Received 26 April 2020; revised 14 June 2021; accepted 29 June 2021; published 16 July 2021)

\begin{abstract}
Electrical control of magnetization is of crucial importance for integrated spintronics devices. Spin-orbit torques (SOT) in heavy-metal/ferromagnetic heterostructures have emerged as a promising tool to achieve efficiently current-induced magnetization reversal. However, the microscopic origin of the SOT is being debated, with the spin Hall effect (SHE) due to nonlocal spin currents and the spin Rashba-Edelstein effect (SREE) due to local spin polarization at the interface being the primary candidates. We investigate the electrically induced out-of-equilibrium spin and orbital polarizations in pure $\mathrm{Pt}$ films and in $\mathrm{Pt} / 3 d$-metal $(\mathrm{Co}, \mathrm{Ni}, \mathrm{Cu})$ bilayer films using ab initio electronic structure methods and linear-response theory. We compute atom-resolved response quantities that allow us to identify the induced spin-polarization contributions that lead to fieldlike (FL) SOTs, mostly associated with the SREE, and dampinglike (DL) SOTs, mostly associated with the SHE, and compare their relative magnitude, dependence on the magnetization direction, as well as their Pt-layer thickness dependence. We find that both the FL and DL components contribute to the resulting SOT at the Pt/Co and $\mathrm{Pt} / \mathrm{Ni}$ interfaces, with the former contributions being larger at the Pt interface layer and the latter larger in the Co or Ni layers. Our calculations show that the electrically induced transverse orbital polarization is exceedingly larger than the induced spin polarization and present even without spin-orbit coupling, in contrast to the spin polarization.
\end{abstract}

DOI: 10.1103/PhysRevMaterials.5.074407

\section{INTRODUCTION}

Electrical control of magnetization has attracted considerable attention because of its potential for high-speed spin-based memories with low-power consumption. Following theoretical predictions $[1,2]$ it was shown that the magnetization of a ferromagnetic layer in a multilayer stack can be switched with a spin-transfer torque (STT) exerted by a spin-polarized electric current flowing through the magnetic layer in perpendicular direction [3-7]. STT enabled the development of current-operated nonvolatile spin-logic devices, such as the STT-magnetic random access memory (STTMRAM) [8]. While STT-based technology is a step forward, there are still shortcomings, such as unintended switching that can occur as the write and read currents flow in the same direction [9].

A different concept to electrical magnetization switching is the more recently discovered spin-orbit torque (SOT) [10-13]. SOT can be observed in a heavy-metal/ferromagnetic bilayer film where the current flows dominantly through the heavy metal and parallel to the ferromagnetic layer. In this configuration, reversible magnetization switching can be achieved in

*leandro.salemi@physics.uu.se

Published by the American Physical Society under the terms of the Creative Commons Attribution 4.0 International license. Further distribution of this work must maintain attribution to the author(s) and the published article's title, journal citation, and DOI. Funded by Bibsam. a very energy efficient way and, moreover, have read and write currents flow in distinct directions through the device [14-17].

While it is evident from experiments that the SOT can be used to efficiently reverse the magnetization in the magnetic layer, its microscopic origin is still to be fully understood. Two candidates for driving the SOT have attracted much attention: the spin Hall effect (SHE) $[18,19]$ and the spin RashbaEdelstein effect (SREE) [20]. Both effects are caused by the spin-orbit interaction, either in the bulk of the material or at an interface, yet their microscopic appearance is drastically different. The SHE is a nonlocal effect wherein an electrical current generates the flow of a transverse spin current to the boundary of the conducting slab (see Refs. [21-24]) where it exerts a torque on the adjacent ferromagnetic layer. The SREE conversely is a local effect: as pointed out by Edelstein [20], a nonequilibrium spin polarization is generated at a symmetry-broken interface by an electric current in the presence of Rashba spin-orbit coupling (SOC) [25]. Both effects have been discussed in the context of SOT switching, in some cases the SHE was considered as the dominant effect $[12,13]$ whereas in other cases the focus was on the SREE $[10,11,14,26]$. In heavy-metal/ferromagnetic bilayer structures, both effects are expected to be present simultaneously and will contribute to the fieldlike (FL) SOT and dampinglike (DL) SOT [27-33], yet their relative contribution remains disputed and continues to be a topic of contemporary investigations [34-36] (see also Ref. [37] for a recent review). First-principles calculations can provide insight in their detailed microscopic origin and offer a way to make a quantitatively comparison $[27,32,33,38-40]$. 
The SHE and SREE are however not the only magnetic effects that can occur. It was discovered theoretically that, in addition to the spin polarization induced by a current through the SHE, also a nonequilibrium orbital polarization can be induced, which represents an orbital Hall effect (OHE) [41-45]. Similarly, the presence of spatial symmetry breaking in a material was recently shown to lead to a significant local orbital polarization, i.e., an orbital Rashba-Edelstein effect (OREE) [46]. Both the OHE and OREE are currently only poorly understood, in terms of their relative magnitudes as well as directions of the induced orbital torques. So far several first-principles calculations have been reported for the OHE [41-45]. A direct observation of the induced orbital polarization is yet to be achieved in experiments (see Refs. [47,48] for recent studies).

In this work, we employ relativistic density functional theory (DFT) and Kubo linear-response theory to compute the spin and orbital response to an external electric field for realistic metallic bilayer structures in which Pt is chosen as the heavy-metal material. Specifically, four different systems are investigated: a pure Pt system and three $\mathrm{Pt} / 3 d$-metal bilayer systems, where the $3 d$ element is $\mathrm{Ni}$, Co or $\mathrm{Cu}$. For these we compute the spin and orbital conductivity and magnetoelectric (ME) tensors resolved for the individual atomic layers in the metallic heterostructures.

In the following, we first introduce the theoretical framework of linear response within DFT and subsequently apply our formalism to compute the spin and orbital responses for the considered bilayer systems, for various Pt thicknesses. We analyze the spatial symmetry of the spin response, which is embodied in the spin ME susceptibility tensor $\chi^{s}$. We show that the direction of the induced spin magnetization $\delta S$ depends on the relative directions of the applied electric field $\boldsymbol{E}$, the equilibrium magnetization direction $\boldsymbol{M}$, and the system geometry. The tensors can be decomposed into odd-in- $\boldsymbol{M}$ and even-in- $\boldsymbol{M}$ components. Based on this parity with respect to $\boldsymbol{M}$, we discuss their relationship to the DL and FL components of the SOT.

The relative importance of those tensor contributions strongly depends on the position of the atomic layer in the slab, and, to a lesser extent, to the thickness of the Pt slab. We investigate furthermore the magnetization-direction dependence of the spin and orbital responses. Whereas the spin response vanishes when the spin-orbit interaction is turned off, off-diagonal components of the orbital ME tensor remain. We compute effective spin-orbit torques on the magnetic $\mathrm{Ni}$ and Co layers and compare our results with previously reported values. Finally, we compare the relative importance of the FL and DL components of $\chi^{S}$ and discuss their relationship to the SHE ad SREE. We find that the induced spin polarization on the $\mathrm{Pt}$ side of the $\mathrm{Pt} / \mathrm{Ni}$ and $\mathrm{Pt} / \mathrm{Co}$ interfaces mainly leads to a FL SOT while the induced spin polarization further into the FM layer contributes more to the DL torque component.

\section{THEORY}

\section{A. Linear response}

The materials are modeled within DFT by the relativistic Kohn-Sham Hamiltonian as implemented in WIEN2K [49],

$$
\hat{H}_{0}|n \boldsymbol{k}\rangle=\epsilon_{n \boldsymbol{k}}|n \boldsymbol{k}\rangle
$$

where $\hat{H}_{0}$ is the relativistic Kohn-Sham Hamiltonian, $|n \boldsymbol{k}\rangle$ the single-electron Kohn-Sham state for band $n$ at wave vector $\boldsymbol{k}$ and $\epsilon_{n k}$ the corresponding eigenenergy. Under the influence of an external perturbation $\hat{V}=-e \hat{\boldsymbol{r}} \cdot \boldsymbol{E}$ where $e$ is the electron charge, $\boldsymbol{E}$ the external electric field and $\hat{\boldsymbol{r}}$ the position operator, the change $\delta \boldsymbol{A}$ in expectation value of a vectorial observable $\mathcal{A}$ associated to vector operator $\hat{\boldsymbol{A}}$, can be expressed within the linear-response formalism $[27,29,30,50]$ as

$$
\delta A_{i}=\sum_{j=x, y, z} \chi_{i j}^{A} E_{j} .
$$

The response $\chi_{i j}^{A}$ is expressed in terms of solutions of $\hat{H}_{0}$,

$$
\begin{aligned}
\chi_{i j}^{A}= & -\frac{i e}{m_{e}} \int_{\Omega} \frac{d \boldsymbol{k}}{\Omega} \sum_{n \neq m} \frac{f_{n \boldsymbol{k}}-f_{m \boldsymbol{k}}}{\hbar \omega_{n m \boldsymbol{k}}} \frac{A_{m n \boldsymbol{k}}^{i} p_{n m \boldsymbol{k}}^{j}}{-\omega_{n m \boldsymbol{k}}+i \tau_{\text {inter }}^{-1}} \\
& -\frac{i e}{m_{e}} \int_{\Omega} \frac{d \boldsymbol{k}}{\Omega} \sum_{n} \frac{\partial f_{n \boldsymbol{k}}}{\partial \epsilon} \frac{A_{n n \boldsymbol{k}}^{i} p_{n n \boldsymbol{k}}^{j}}{i \tau_{\text {intra }}^{-1}}
\end{aligned}
$$

with $m_{e}$ the mass of the electron, $f_{n k}$ the occupation of Kohn-Sham state $|n \boldsymbol{k}\rangle, \Omega$ the Brillouin-zone volume, $p_{n m \boldsymbol{k}}^{j}$ the

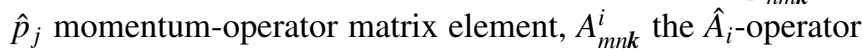
matrix element and $\hbar \omega_{n m k}=\epsilon_{n k}-\epsilon_{m k}$, the difference of Kohn-Sham eigenergies. As discussed below, we use for $\hat{A}$ the spin and orbital angular momentum operators, $\hat{\boldsymbol{S}}$ and $\hat{\boldsymbol{L}}$, as well as the spin and orbital current-density operators, $\hat{J}^{S}$ and $\hat{\boldsymbol{J}}^{L}$. The product $J_{m n k}^{i S} p_{n m k}^{j}$ is proportional to the spin Berry connection [48]. The quantity $\tau_{\text {inter }}\left(\tau_{\text {intra }}\right)$ is the electronic lifetime for inter (intra) band transitions. In the main part of this work, $\tau_{\text {inter }}$ and $\tau_{\text {intra }}$ are set to $\hbar \tau_{\text {inter }}^{-1}=0.272 \mathrm{eV}$ and $\hbar \tau_{\text {intra }}^{-1}=0.220 \mathrm{eV}$. Those values have been determined by comparing linear-response calculations to experimental conductivity data for Pt thin films [51]. The influence of the electronic lifetime on the computed nonequilibrium spin and orbital polarizations is discussed in Sec. III A 4.

\section{B. Angular momentum and flow of angular momentum}

The induced angular momentum is composed of a spin and orbital contribution. Let us first focus on the spin part.

The spin operator $\hat{\boldsymbol{S}}$ and spin-density current operator $\hat{\boldsymbol{J}}^{S_{k}}$ can be defined as

$$
\begin{gathered}
\hat{\boldsymbol{S}}=\frac{\hbar}{2}\left(\hat{\sigma}_{x}, \hat{\sigma}_{y}, \hat{\sigma}_{z}\right), \\
\hat{\boldsymbol{J}}^{S_{k}}=\frac{\left\{\hat{S}_{k}, \hat{\boldsymbol{p}}\right\}}{2 m_{e} V},
\end{gathered}
$$

where $\hat{\sigma}_{x}, \hat{\sigma}_{y}$, and $\hat{\sigma}_{z}$ are the Pauli matrices, $\{.,$.$\} denotes the$ anti-commutator, $V$ is a reference volume and $k(k=x, y, z)$ an index specifying the direction of the spin polarization carried by the spin-current density. In this work, $V$ refers to the individual atomic spheres, allowing us to compute atomprojected quantities (see Appendix A for details).

Using the linear-response formalism, we can compute the out-of-equilibrium electrically induced spin angular momentum $\delta \boldsymbol{S}$ as well as the induced spin-current density $\boldsymbol{J}^{S_{k}}$, using

$$
\begin{gathered}
\delta \boldsymbol{S}=\boldsymbol{\chi}^{S} \boldsymbol{E}, \\
\boldsymbol{J}^{S_{k}}=\boldsymbol{\sigma}^{S_{k}} \boldsymbol{E},
\end{gathered}
$$




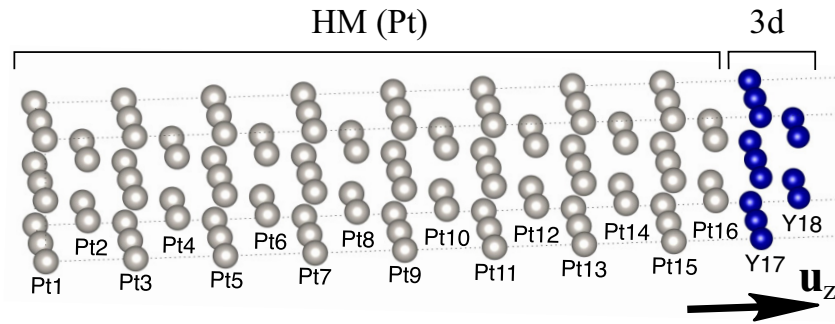

FIG. 1. Schematic of a typical system studied in this work, a $n \mathrm{Pt} / 2 Y$ bilayer. There are $n(=16$, here $)$ monolayers of Pt heavy metal (HM) capped by two $Y$ monolayers, where $Y$ is $\mathrm{Ni}$, Co, or nonmagnetic $\mathrm{Cu}$ or Pt. The $z$ axis is taken normal to the slab, with unit vector $\boldsymbol{u}_{z}$. Each atomic plane is numbered with an index, where index 1 refers to the Pt atomic-layer interfaced with vacuum, $n$ to the $\mathrm{Pt}$ atomic-layer interfaced with the $3 d$ element in layer $n+1$, and $n+2$ labels the top layer at the vacuum interface.

where $\chi^{S}$ is the spin ME susceptibility tensor and $\boldsymbol{\sigma}^{S_{k}}$ the spin conductivity tensor. Both $\chi^{S}$ and $\boldsymbol{\sigma}^{S_{k}}$ are real 2 nd-rank tensors, but note that, due to the spin component dependence, the spin conductivity tensor can be associated with a 3rd-rank tensor, $\sigma^{S}$.

Analogous quantities can be straightforwardly defined for the orbital angular momentum $\hat{\boldsymbol{L}}$. Thus, we can define the orbital ME susceptibility tensor $\chi^{L}$ and orbital conductivity tensor $\sigma^{L_{k}}$,

$$
\begin{aligned}
\delta \boldsymbol{L} & =\chi^{L} \boldsymbol{E}, \\
\boldsymbol{J}^{L_{k}} & =\boldsymbol{\sigma}^{L_{k}} \boldsymbol{E},
\end{aligned}
$$

where $\delta \boldsymbol{L}$ is the out-of-equilibrium electrically induced orbital angular momentum and $\boldsymbol{J}^{L_{k}}$ the induced orbital current density.

\section{Computational methodology}

The bilayer structures that are studied here consist of several $\mathrm{Pt}$ monoatomic layers that are covered with two monoatomic layers of the $3 d$ elements $\mathrm{Ni}, \mathrm{Co}$ or $\mathrm{Cu}$ (see Fig. 1). For comparison, we also study the pure Pt system, where the top two monolayers consist of Pt. The nomenclature used in this paper is the following: we denote our systems by $n \mathrm{Pt} / 2 Y$ where $n$ is the total number of Pt monolayers and $Y$ is either $\mathrm{Ni}, \mathrm{Co}, \mathrm{Cu}$, or $\mathrm{Pt}$. The minimum total number of Pt monolayers used in our calculations is 2 while the maximum is 18 (denoted as $16 \mathrm{Pt} / 2 \mathrm{Pt}$ ). The maximum thickness achieved is then $\sim 3.2 \mathrm{~nm}$. The direction normal to the interfaces is taken as the $z$ axis. The monoatomic layers are labeled from $z=1$ for the Pt monoatomic layer at the interface with vacuum (leftmost layer in Fig. 1) to $z=n+2$ for the $Y$ monoatomic layer at the interface with vacuum (rightmost layer in Fig. 1). Particular positions can be identified, like $z=n$ for the Pt monoatomic layer at the $\mathrm{Pt} / Y$ interface and $z=n+1$ for the $Y$ monoatomic layer at the $\mathrm{Pt} / Y$ interface.

To compute the spin and orbital susceptibility and conductivity tensors, we use the following three-step procedure. (1) The cell parameters and atomic positions of the heterostructures are fully relaxed with the DFT package SIESTA [52]. (2) Using the relaxed atomic positions, the ground-state
Kohn-Sham wave functions and energies are self-consistently computed with the accurate full-potential, all-electron DFT package WIEN2K [49]. (3) Using the relativistic Kohn-Sham wave functions and energies, we compute the response tensors defined by Eq. (3).

As the DFT packages used employ full 3D periodic boundary conditions, all heterostructures contain $20 \AA$ of vacuum to avoid spurious interactions with neighboring simulation cells. More details on the computational recipe are given in Appendix A.

\section{Symmetry considerations}

\section{Symmetry of the ME tensors}

The structures studied in this work are tetragonal, with nonmagnetic point group $4 \mathrm{~mm}$. The response tensor $\chi^{S}$ can be expanded as

$$
\chi_{i j}^{S}=\chi_{i j}^{(0) S}+\sum_{k} \chi_{i j k}^{(1) S} \mathcal{M}_{k}+\sum_{k l} \chi_{i j k l}^{(2) S} \mathcal{M}_{k} \mathcal{M}_{l}+\ldots,
$$

where $\mathcal{M}_{k}$ is the $k$ th component of the unit vector of magnetization, i.e., $\boldsymbol{M}=|\boldsymbol{M}| \boldsymbol{M}$, with $\boldsymbol{M}$ the equilibrium magnetization. As detailed in Ref. [53], for $\boldsymbol{M} \| \boldsymbol{u}_{z}, \chi^{S}$ can be written as

$$
\chi^{S}=\left(\begin{array}{ccc}
\chi_{x x}^{S} & \chi_{x y}^{S} & 0 \\
\chi_{y x}^{S} & \chi_{y y}^{S} & 0 \\
0 & 0 & \chi_{z z}^{S}
\end{array}\right)\left[\boldsymbol{M} \| \boldsymbol{u}_{z}\right],
$$

where $\chi_{x y}^{S}=-\chi_{y x}^{S}$ and $\chi_{x x}^{S}=\chi_{y y}^{S} \neq \chi_{z z}^{S}$. With $\boldsymbol{M}$ out of plane, the system exhibits an in-plane $x / y$ spatial symmetry, which is fully recovered in our calculations. The $\chi^{S}$ tensor can be further decomposed into odd-in- $\boldsymbol{M}$ and even-in- $\boldsymbol{M}$ components,

$$
\chi^{S}(\boldsymbol{M})=\chi_{o}^{S}(\boldsymbol{M})+\chi_{e}^{S}(\boldsymbol{M})
$$

with specifically,

$$
\begin{aligned}
\chi_{e}^{S} & =\left(\begin{array}{ccc}
0 & \chi_{x y}^{S} & 0 \\
\chi_{y x}^{S} & 0 & 0 \\
0 & 0 & 0
\end{array}\right), \\
\chi_{o} & =\left(\begin{array}{ccc}
\chi_{x x}^{S} & & 0 \\
0 & \chi_{y y}^{S} & 0 \\
0 & 0 & \chi_{z z}^{S}
\end{array}\right) .
\end{aligned}
$$

A nonzero odd-in- $\boldsymbol{M}$ part can obviously not exist for nonmagnetic systems $(n \mathrm{Pt} / 2 \mathrm{Pt}$ and $n \mathrm{Pt} / 2 \mathrm{Cu})$, which is as well recovered in our calculations. The spin response is highly dependent on the magnetization direction. Setting the magnetization in plane, $\boldsymbol{M} \| \boldsymbol{u}_{x}$, the $\chi^{S}$ tensor can be written as

$$
\chi^{S}=\left(\begin{array}{ccc}
0 & \chi_{x y}^{S} & \chi_{x z}^{S} \\
\chi_{y x}^{S} & 0 & 0 \\
\chi_{z x}^{S} & 0 & 0
\end{array}\right)\left[\boldsymbol{M} \| \boldsymbol{u}_{x}\right],
$$

being different from the $\boldsymbol{M} \| \boldsymbol{u}_{z}$ case. Now the $\chi_{x y}, \chi_{y x}$ elements are even-in- $\boldsymbol{M}$ and the $\chi_{x z}, \chi_{z x}$ elements odd-in- $\boldsymbol{M}$. We can furthermore mention already that the orbital $\chi^{L}$ tensor has the same nonzero elements with the same $\boldsymbol{M}$ parity. Note 
that the magnetization direction breaks the $x / y$ symmetry such that the $x y$ and $y x$ components of $\chi^{S / L}$ may be different. Such difference, if relevant, comes from terms with powers of $\mathcal{M}$ of order higher than two in Eq. (10). As we will see later, those higher order corrections are indeed relevant for the spin response. The symmetry of the system is naturally incorporated in the electronic density $n(\boldsymbol{r})$ and magnetization density $\boldsymbol{m}(\boldsymbol{r})$ computed with DFT. As a result, our calculations, in which we do not impose any symmetry constraints, provide the full $\boldsymbol{M}$-dependent response, i.e., the left hand-side of Eq. (10).

Depending on the relative orientation of the induced spin polarization $\delta \boldsymbol{S}$ with respect to (1) the applied electric field $\boldsymbol{E}$, (2) the normal direction $\boldsymbol{u}_{z}$ and (3) the equilibrium magnetization vector $\boldsymbol{M}$, the components of the ME susceptibility $\chi^{S}$ can be classified according to three categories.

(1) $\boldsymbol{E}$-transverse components $\left(\boldsymbol{E}_{\perp}\right)$ :

$$
\delta \boldsymbol{S} \propto \boldsymbol{E} \times \boldsymbol{u}_{z} .
$$

(2) $\boldsymbol{M}$-transverse components $\left(\boldsymbol{M}_{\perp}\right)$ :

$$
\delta \boldsymbol{S} \propto\left(\boldsymbol{E} \times \boldsymbol{u}_{z}\right) \times \boldsymbol{M} .
$$

(3) $\boldsymbol{M}$-longitudinal component $\left(\boldsymbol{M}_{\|}\right)$:

$$
\delta \boldsymbol{S} \propto \boldsymbol{M} \text { when } \boldsymbol{E} \| \boldsymbol{u}_{z} .
$$

\section{Symmetry of the SOT}

The SOT $\mathcal{T}$ felt by the equilibrium magnetization $\boldsymbol{M}$ can be written as

$$
\mathcal{T}=\boldsymbol{M} \times \delta \boldsymbol{B},
$$

where $\delta \boldsymbol{B}$ is the SOT effective magnetic field, which originates from the electrically induced change in the exchangecorrelation effective field $\boldsymbol{B}_{\text {XC. }}$. We can approximate $\delta \boldsymbol{B}$ as

$$
\delta \boldsymbol{B} \approx\left|\boldsymbol{B}_{\mathrm{XC}}\right| \frac{\delta \boldsymbol{S}}{|\boldsymbol{S}|},
$$

where $S$ is the equilibrium value of the expectation value of $\hat{\boldsymbol{S}}$. Essentially, Eq. (19) assumes that the direction of the exchange-correlation effective field changes while its strength is unaffected. Note that because the spatial distribution of $\delta \boldsymbol{S}$ may differ for different orbital characters (e.g., $s, p, d$ ), Eq. (19) is an estimate of $\delta \boldsymbol{B}$.

Using $\boldsymbol{M}=-2 \mu_{B} \boldsymbol{S}$, where $\mu_{B}$ is the Bohr magneton, and Eq. (19), the torque can be evaluated as

$$
\mathcal{T}=-2 \mu_{B}\left|\boldsymbol{B}_{\mathrm{XC}}\right|(\mathcal{M} \times \delta \boldsymbol{S})
$$

where we used that $\mathcal{M}=\boldsymbol{S} /|\boldsymbol{S}|$. With the induced spin polarization [Eq. (6)] this can be written as

$$
\mathcal{T}=-2 \mu_{B}\left|\boldsymbol{B}_{\mathrm{XC}}\right|\left[\mathcal{M} \times\left(\boldsymbol{\chi}^{S} \boldsymbol{E}\right)\right] .
$$

Since $\chi^{S}$ can be decomposed in odd-in- $\boldsymbol{M}$ and even-in- $\boldsymbol{M}$ components, we can always decompose the SOT in an odd (o) and an even (e) component,

$$
\mathcal{T}=\mathcal{T}_{\mathrm{o}}+\mathcal{T}_{\mathrm{e}}
$$

We can compute $\mathcal{T}_{\mathrm{o}}$ and $\mathcal{T}_{\mathrm{e}}$, e.g. for an in-plane field, $\boldsymbol{E}=$ $|\boldsymbol{E}| \boldsymbol{u}_{x}$. When $\boldsymbol{M}|| \boldsymbol{u}_{z}$, using Eq. (13), we obtain

$$
\begin{aligned}
& \mathcal{T}_{\mathrm{o}}=+2 \mu_{B}\left|\boldsymbol{B}_{\mathrm{XC}}\right||\boldsymbol{E}| \chi_{y x}^{S} \boldsymbol{u}_{x}, \\
& \mathcal{T}_{\mathrm{e}}=-2 \mu_{B}\left|\boldsymbol{B}_{\mathrm{XC}}\right||\boldsymbol{E}| \chi_{x x}^{S} \boldsymbol{u}_{y},
\end{aligned}
$$

TABLE I. The $\boldsymbol{E}$-transverse $\left(\boldsymbol{E}_{\perp}\right), \boldsymbol{M}$-transverse $\left(\boldsymbol{M}_{\perp}\right)$, and $\boldsymbol{M}$ longitudinal $\left(\boldsymbol{M}_{\|}\right)$components of the $\chi$ tensor for $\boldsymbol{M}$ parallel to $\boldsymbol{u}_{z}$, $\boldsymbol{u}_{x}$, or $\boldsymbol{u}_{y}$. Each row summarizes the equivalency of the $\chi$ components for the three magnetization directions. The spatial symmetry of the induced spin polarization $\delta \boldsymbol{S}$ and whether it leads to a dampinglike or fieldlike spin-orbit torque $\mathcal{T}$ are also provided.

\begin{tabular}{cccccc}
\hline \hline & $\boldsymbol{M} \| \boldsymbol{u}_{z}$ & $\boldsymbol{M} \| \boldsymbol{u}_{x}$ & $\boldsymbol{M} \| \boldsymbol{u}_{y}$ & $\delta \boldsymbol{S}$ dependence & $\mathcal{T}$ \\
\hline $\boldsymbol{E}_{\perp}$ & $\chi_{x y / y x}$ & $\chi_{x y / y x}$ & $\chi_{x y / y x}$ & $\boldsymbol{E} \times \boldsymbol{u}_{z}$ & $\mathrm{FL}$ \\
$\boldsymbol{M}_{\perp}$ & $\chi_{x x / y y}$ & $\chi_{z x}$ & $\chi_{z y}$ & $\boldsymbol{M} \times\left(\boldsymbol{E} \times \boldsymbol{u}_{z}\right)$ & $\mathrm{DL}$ \\
$\boldsymbol{M}_{\|}$ & $\chi_{z z}$ & $\chi_{x z}$ & $\chi_{y z}$ & $\boldsymbol{M}\left(\boldsymbol{E} \| \boldsymbol{u}_{z}\right)$ & - \\
\hline \hline
\end{tabular}

while for $\boldsymbol{M} \| \boldsymbol{u}_{x}$ this yields [see Eq. (14)]

$$
\begin{aligned}
& \mathcal{T}_{\mathrm{o}}=-2 \mu_{B}\left|\boldsymbol{B}_{\mathrm{XC}}\right||\boldsymbol{E}| \chi_{y x}^{S} \boldsymbol{u}_{x}, \\
& \mathcal{T}_{\mathrm{e}}=+2 \mu_{B}\left|\boldsymbol{B}_{\mathrm{XC}}\right||\boldsymbol{E}| \chi_{z x}^{S} \boldsymbol{u}_{y} .
\end{aligned}
$$

We can now easily establish the link between the symmetry of the components of $\boldsymbol{\chi}^{S}$, their evenness or oddness in $\boldsymbol{M}$, and the commonly discussed fieldlike torque $\mathcal{T}_{\text {FL }}$ and dampinglike torque $\mathcal{T}_{\text {DL }}$, specifically,

$$
\begin{aligned}
& \mathcal{T}_{\mathrm{FL}} \propto \boldsymbol{M} \times\left(\boldsymbol{E} \times \boldsymbol{u}_{z}\right), \\
& \mathcal{T}_{\mathrm{DL}} \propto \boldsymbol{M} \times\left[\boldsymbol{M} \times\left(\boldsymbol{E} \times \boldsymbol{u}_{z}\right)\right] .
\end{aligned}
$$

We recognize that $\mathcal{T}_{\mathrm{o}}$, and therefore the $\boldsymbol{E}_{\perp}$ components, correspond to $\mathcal{T}_{\text {FL }}$ while $\mathcal{T}_{\mathrm{e}}$, and therefore the $\boldsymbol{M}_{\perp}$ components, correspond to $\mathcal{T}_{\text {DL }}$.

A similar classification can be carried out for the cases where $\boldsymbol{M} \| \boldsymbol{u}_{x}$ and $\boldsymbol{M} \| \boldsymbol{u}_{y}$. The classification and symmetry relations for these magnetization directions are summarized for convenience in Table I.

\section{RESULTS}

\section{A. Spin response}

\section{Magnetization out of plane}

We start with the case where $\boldsymbol{M} \| \boldsymbol{u}_{z}$. It is instructive to consider first the thickest heterostructures, i.e., $16 \mathrm{Pt} / 2 \mathrm{Ni}$, $16 \mathrm{Pt} / 2 \mathrm{Co}, 16 \mathrm{Pt} / 2 \mathrm{Cu}$, and $16 \mathrm{Pt} / 2 \mathrm{Pt}$. In Figs. 2(a), 2(b) and 2(c), we show the computed atomic layer-resolved profiles of the aforementioned nonzero components of $\chi^{S}$, as well as related components of the spin conductivity tensor $\sigma_{z x}^{S_{y}}, \sigma_{z x}^{S_{x}}$, and $\sigma_{z z}^{S_{z}}$ in Figs. 2(d), 2(e) and 2(f), respectively.

In all cases, we observe that the response of the Pt atomic layer at the vacuum interface $(z=1)$ is virtually independent on the type of $Y$ atom used, suggesting that these systems are thick enough to isolate the $\mathrm{Pt} / 3 d$-interface properties. The inclusion of the two monoatomic layers of $3 d$ elements mainly impacts the $\chi^{S}$ and $\sigma^{S}$ profiles close to their interface.

For the $\boldsymbol{E}$-transverse components [Figs. 2(a) and 2(d)], both $\chi^{S}$ and $\sigma^{S}$ are qualitatively barely impacted in the bulk $\mathrm{Pt}$ region by the replacement of the two last $\mathrm{Pt}$ atomic monolayers by two $3 d$ atomic monolayers. The profile of $\sigma_{z x}^{S_{y}}$ is in all cases mostly defined by a plateau in the center of the $\mathrm{Pt}$ layer. This specific component of $\boldsymbol{\sigma}^{S}$ is customarily identified with the SHE in Pt-bulk calculations. The spin-accumulation profile related to $\chi_{y x}^{S}$ across the Pt layer strongly resembles 

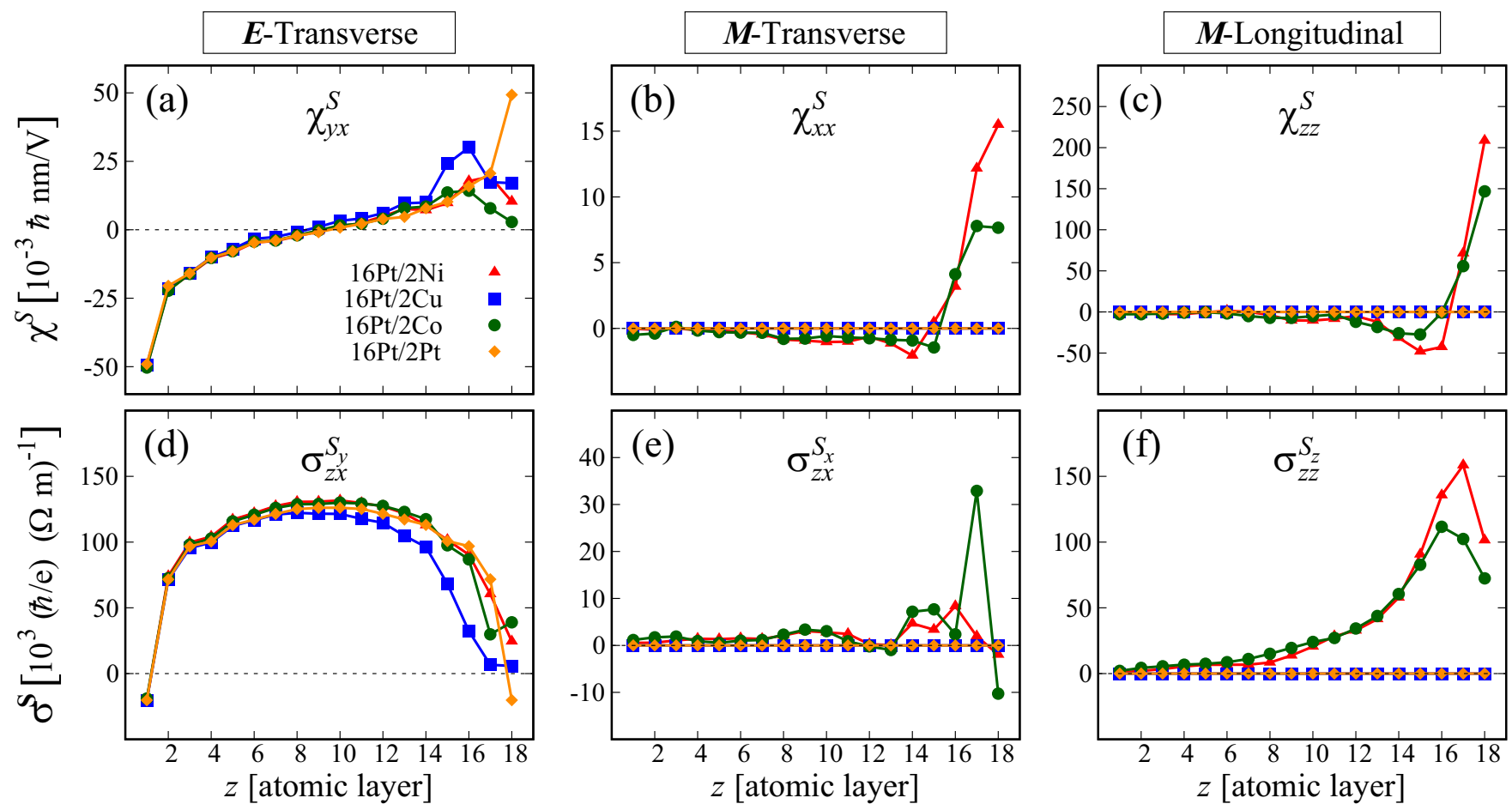

FIG. 2. Computed atomic layer-resolved nonzero components of the spin ME susceptibility $\chi^{S}$ and spin conductivity $\boldsymbol{\sigma}^{S}$ of the $16 \mathrm{Pt} / 2 Y$ structures. (a) The $\boldsymbol{E}$-transverse component $\chi_{y x}^{S}$, (b) $\boldsymbol{M}$-transverse component $\chi_{x x}^{S}$, and (c) $\boldsymbol{M}$-longitudinal component $\chi_{z z}^{S}$. The corresponding components of the spin conductivity tensor are given as (d) $\boldsymbol{E}$-transverse $\sigma_{z x}^{S_{y}}$, (e) $\boldsymbol{M}$-transverse $\sigma_{z x}^{S_{x}}$, and (f) $\boldsymbol{M}$-longitudinal $\sigma_{z z}^{S_{z}}$. The $\boldsymbol{E}$ transverse components of $\boldsymbol{\sigma}^{S}$ can be associated with SHE. The $\boldsymbol{M}$-transverse components of $\chi$ are nonzero only for magnetic systems (16Pt/2Ni and $16 \mathrm{Pt} / 2 \mathrm{Co}$ ) and in the vicinity of the interface, suggesting the importance of spin-splitting of the electronic states. The $\boldsymbol{M}$-longitudinal components are discussed in the text. See Fig. 1 for the numbering of the atomic layers.

the type spin accumulation that is expected from transverse spin flow. Reversing the magnetization of the Ni and Co layers from $+\boldsymbol{u}_{z}$ to $-\boldsymbol{u}_{z}$ in the calculations, moreover, does not have a notable effect on the ( $\boldsymbol{M}$-even) spin accumulation given by $\chi_{y x}^{S}$. These results are in agreement with what is observed in SHE calculations for bulk Pt [51,54]. Note that the accumulated spin moment is given by $\delta \boldsymbol{M}=-2\left(\mu_{B} / \hbar\right) \delta \boldsymbol{S}$.

For the $16 \mathrm{Pt} / 2 \mathrm{Cu}$ system, the discussion cannot be performed on the basis of torques ( $\boldsymbol{M}$ is ill-defined). For the spin accumulation related to $\chi_{y x}^{S}$, compared to the pure $\mathrm{Pt}$ case, we observe an increase of accumulated spin density at the $\mathrm{Pt}$ side of the $\mathrm{Pt} / \mathrm{Cu}$ interface as well as a decrease of spin density within the $\mathrm{Cu}$ layer. For $16 \mathrm{Pt} / 2 \mathrm{Ni}$ and $16 \mathrm{Pt} / 2 \mathrm{Co}$, one can in addition observe that a spin depolarization occurs in the two ferromagnetic layers, as has been discussed in Refs. [55-59].

The $\boldsymbol{M}_{\perp}$ components [Figs. 2(b) and 2(e)], are nonexistent for nonmagnetic pure $\mathrm{Pt}$ and $\mathrm{Pt} / \mathrm{Cu}$ system. Sizable values are only obtained close to the interface with $\mathrm{Co}$ and $\mathrm{Ni}$, both for $\chi^{S}$ and $\boldsymbol{\sigma}^{S}$. Remarkably, while the $\boldsymbol{E}_{\perp}$ and $\boldsymbol{M}_{\perp}$ components are comparable in size close to the interface, their features differ greatly: (1) there is no bulklike behavior for $\sigma_{z y}^{S_{y}} / \sigma_{z x}^{S_{x}},(2)$ those components are nonexistent in bulk Pt, and (3) they are magnetization direction dependent. Although there is a spatial symmetry breaking at the $\mathrm{Pt} / \mathrm{Cu}$ interface, no spin polarization is induced, which strongly suggests the importance of spin-split electronic states at the interface for $\chi_{x x}^{S}$ and $\sigma_{z x}^{S_{x}}$. The odd-in- $\boldsymbol{M}$ spin conductivity $\sigma_{z x}^{S_{x}}$ was recently named magnetic SHE [60], to distinguish it from the conventional SHE $\sigma_{z x}^{S_{y}}$ that is even-in- $\boldsymbol{M}$ [61].

As aforementioned, the $\boldsymbol{M}$-longitudinal components [see Figs. 2(c) and 2(f)] are peculiar in the sense that they are not SOT-related (the usual SOT configuration does not involve out-of-plane electrical fields and, also, no torque is generated by a spin accumulation parallel to the static moment). Although such components can in principle be obtained via a symmetry analysis (cf. Ref. [53]), they have not been, to the best of our knowledge, investigated theoretically so far. As will be clarified further below, this effect is due to the spin-orbit interaction. Here, an electric field applied parallel to the out-of-plane magnetization causes a sign-changing spin polarization along $\boldsymbol{M}$ in the $\sim 5$ topmost monolayers. This is clearly a magnetic effect, as it does not exist for the nonmagnetic systems. The spin conductivity $\sigma_{z z}^{S_{z}}$ shows a decaying behavior from $z=16$ to 1 , but this decay is slower than that of the equilibrium spin magnetization in the systems. Also, similar to the $\boldsymbol{M}$-transverse component, no "bulklike" behavior is observed. A possible way of observing this previously unidentified SOC-induced effect could be achieved by gating the ferromagnetic layer from the top and monitor a change of its magnetization.

So far, we focused on the components of the spin conductivity tensor giving rise to spin currents flowing along $\boldsymbol{u}_{z}$. While those components are the ones that should be of interest 


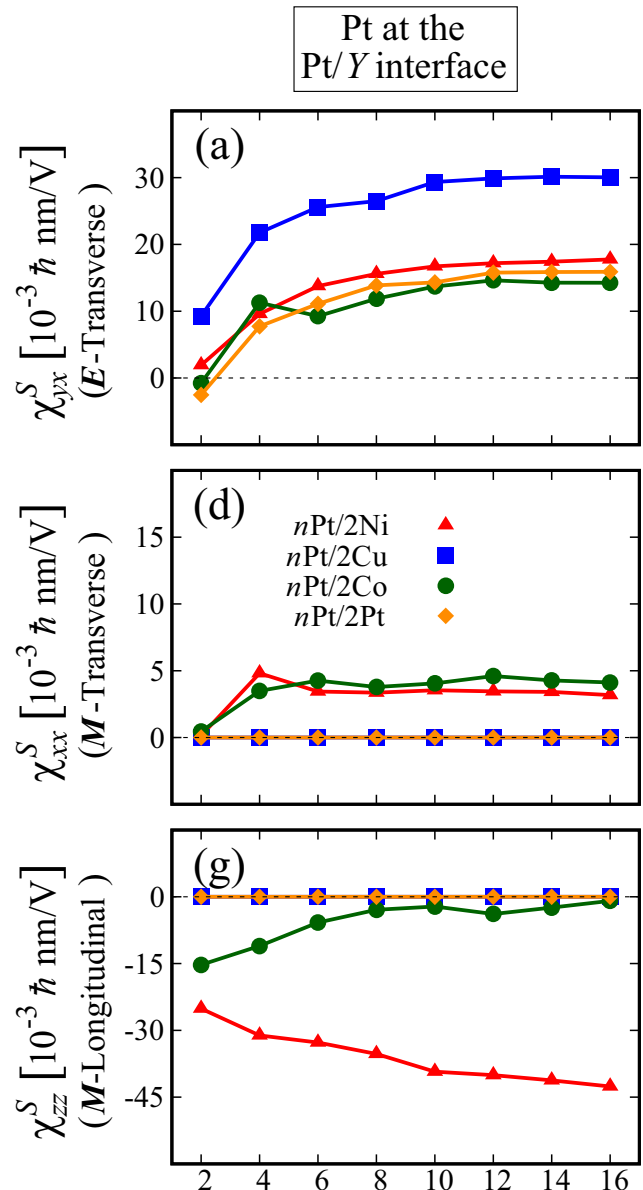

Number of Pt monolayers $n$
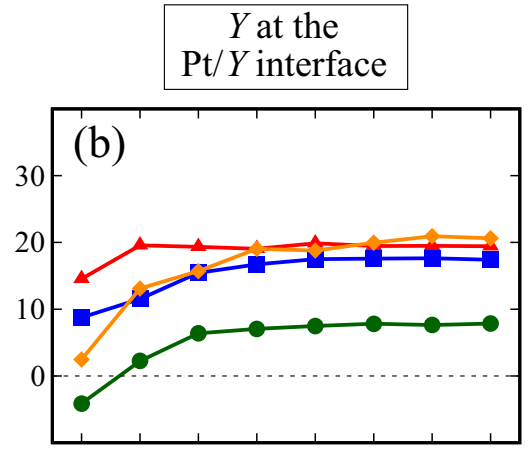

(e)
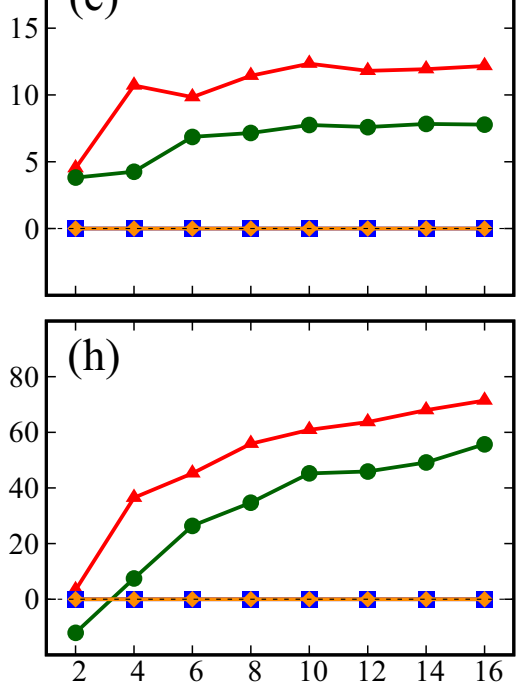

Number of Pt monolayers $n$
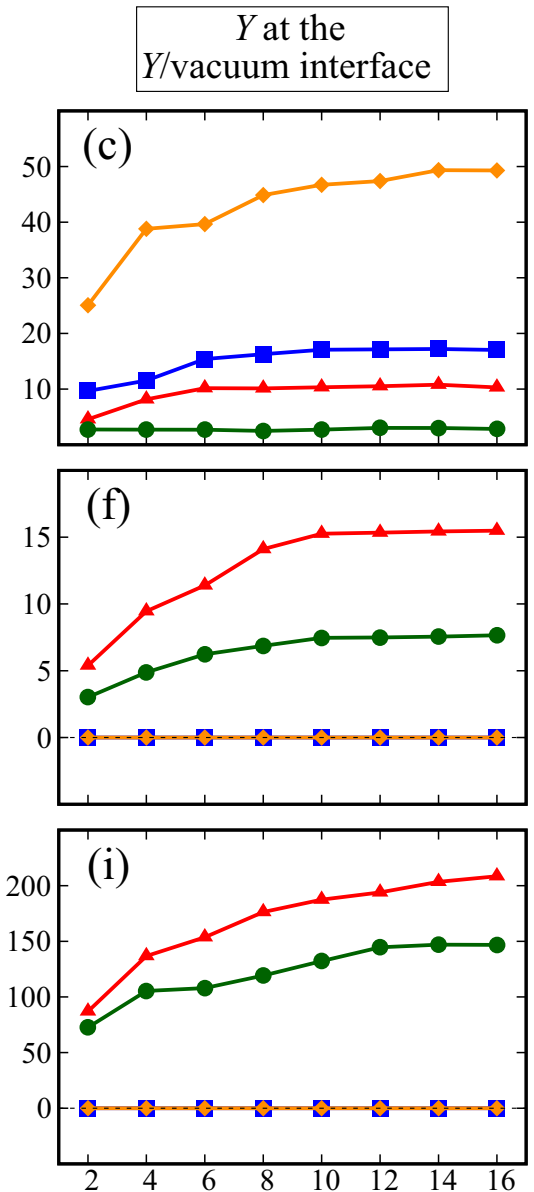

Number of Pt monolayers $n$

FIG. 3. Dependence of the spin ME susceptibility tensor $\chi^{S}$ on the number of Pt monolayers $n$. (Top raw) $\boldsymbol{E}$-transverse component of $\chi^{S}$, for (a) the $\mathrm{Pt}$ atom at the $\mathrm{Pt} / Y$ interface, (b) the $Y$ atom at the $\mathrm{Pt} / Y$ interface, and (c) the $Y$ atom at the $Y /$ vacuum interface. (Second row) $\boldsymbol{M}$-transverse component of $\chi^{S}$, for (d) the Pt atom at the $\mathrm{Pt} / Y$ interface, (e) the $Y$ atom at the Pt $/ Y$ interface, and (f) the $Y$ atom at the $Y$ /vacuum interface. (Bottom row) $M$-longitudinal component of $\chi^{S}$, for (g) the $\mathrm{Pt}$ atom at the $\mathrm{Pt} / Y$ interface, (h) the $Y$ atom at the $\mathrm{Pt} / Y$ interface, and (i) the $Y$ atom at the $Y$ /vacuum interface.

for understanding SOT in bilayer structures, other nonzero components can be observed, as well. For a magnetic system with the $4 m m$ point group, with $\mathcal{M} \| \boldsymbol{u}_{z}$, we find that $\boldsymbol{\sigma}^{S}$ can be written as

$$
\begin{aligned}
\boldsymbol{\sigma}^{S_{x}} & =\left(\begin{array}{ccc}
0 & 0 & \sigma_{x z}^{S_{x}} \\
0 & 0 & \sigma_{y z}^{S_{x}} \\
\sigma_{z x}^{S_{x}} & \sigma_{z y}^{S_{x}} & 0
\end{array}\right), \\
\boldsymbol{\sigma}^{S_{y}} & =\left(\begin{array}{ccc}
0 & 0 & \sigma_{x z}^{S_{y}} \\
0 & 0 & \sigma_{y z}^{S_{y}} \\
\sigma_{z x}^{S_{y}} & \sigma_{z y}^{S_{y}} & 0
\end{array}\right), \\
\boldsymbol{\sigma}^{S_{z}} & =\left(\begin{array}{ccc}
\sigma_{x x}^{S_{z}} & \sigma_{x y}^{S_{z}} & 0 \\
\sigma_{y x}^{S_{z}} & \sigma_{y y}^{S_{z}} & 0 \\
0 & 0 & \sigma_{z z}^{S_{z}}
\end{array}\right) .
\end{aligned}
$$

The components associated to the SHE, i.e., $\sigma_{i j}^{S_{k}}$, where the indices are such that $\epsilon_{i j k} \neq 0\left(\epsilon_{i j k}\right.$ is the Levi-Civita symbol), are nonzero whether the system is magnetic or not. However, while in cubic systems like bulk Pt they are all equal in magnitude, here, because of the symmetry breaking with respect to the $z$ axis, the tensor elements are not invariant under exchange of $z$ and $x$ or $y$ indices. The other seven components, that is $\sigma_{x z}^{S_{x}}, \sigma_{z x}^{S_{x}}, \sigma_{y z}^{S_{y}}, \sigma_{z y}^{S_{y}}, \sigma_{x x}^{S_{z}}, \sigma_{y y}^{S_{z}}$, and $\sigma_{z z}^{S_{z}}$ only exists for magnetic systems. Some of those magnetic components have been discussed recently [61-65].

\section{Pt-thickness dependence}

Next, we investigate the Pt layer thickness dependence of the $\boldsymbol{E}_{\perp}, \boldsymbol{M}_{\perp}$, and $\boldsymbol{M}_{\|}$components of $\chi^{S}$. The number of $\mathrm{Pt}$ monolayers for our $n \mathrm{Pt} / 2 Y$ systems is varied from $n=2$ (Pt thickness $\sim 0.38 \mathrm{~nm}$ ) to $n=16$ (Pt thickness $\sim 3.08 \mathrm{~nm}$ ). Figure 3 shows the computed Pt-thickness dependence where each column of the figure focuses on one particular atomic monolayer, with, from left to right, the $\mathrm{Pt}$ monolayer at the $\mathrm{Pt} / Y$ interface, the $Y$ monolayer at the $\mathrm{Pt} / Y$ interface, and the $Y$ atomic monolayer at the $Y /$ vacuum interface. Each row focuses on one particular component, namely, from top to bottom, the $\boldsymbol{E}_{\perp}, \boldsymbol{M}_{\perp}$, and $\boldsymbol{M}_{\|}$components. The values of the tensor elements that give rise to the SOT, the $\boldsymbol{E}_{\perp}$ and $\boldsymbol{M}_{\perp}$ components, barely fluctuate beyond $n=8$ (Pt thickness $\geqslant 1.54 \mathrm{~nm}$ ). 


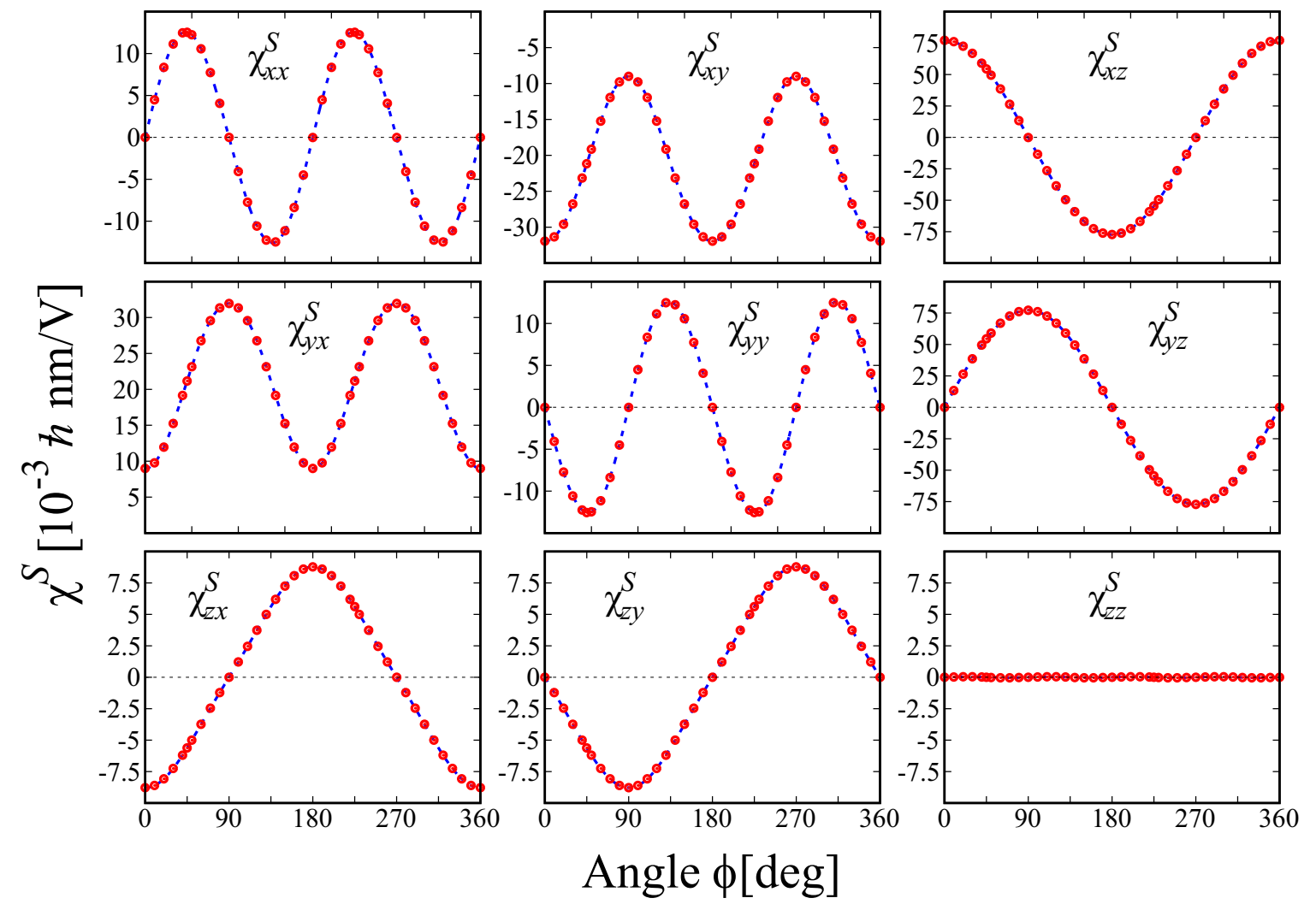

FIG. 4. Computed angular dependence of the spin ME tensor $\chi^{S}$ of the $12 \mathrm{Pt} / 2 \mathrm{Co}$ system at the first Co atom for in-plane magnetizations, given as a function of the azimuthal angle $\phi$.

Thus both $\mathcal{T}_{\text {FL }}$ and $\mathcal{T}_{\text {DL }}$ approach their maximum values already for relatively thin Pt layers, consistent with recent investigations $[33,66]$.

For the $\boldsymbol{E}_{\perp}$ components, in the case of pure $\mathrm{Pt}(n \mathrm{Pt} / 2 \mathrm{Pt})$, $\chi_{y x}^{S}$ tends to increase the closer we come to the last layer, which is typically what we would expect from a transportgenerated spin accumulation profile. When the two last layers are replaced by a magnetic element $(Y=\mathrm{Co}$ or Ni) drastic changes occur. First, we observe that $\chi_{y x}^{S}$ is bigger for the $3 d$ monolayer closer to the Pt layer than for the second $Y$ layer. Second, at a fixed position, $\chi_{y x}^{S}$ is bigger for $Y=\mathrm{Ni}$ than $Y=$ Co.

For the $\boldsymbol{M}_{\perp}$ components, associated to $\mathcal{T}_{\mathrm{DL}}$, the $\chi_{x x}^{S}$ for the $\mathrm{Pt}$ monolayer at the $\mathrm{Pt} / Y$ interface [Fig. 3(d)] is virtually identical for $Y=\mathrm{Ni}$ or $\mathrm{Co}$ and for all $\mathrm{Pt}$ thicknesses considered. In the first and second magnetic monolayers [Figs. 3(e)) and 3(f)], the $\chi_{x x}^{S}$ is, in both cases, bigger for $Y=\mathrm{Ni}$ than $Y=$ Co. The bottom row, lastly, shows the $\boldsymbol{M}$-longitudinal spin accumulation. Also here, we obtain that a larger magnitude of $\chi_{z z}^{S}$ is generated for $Y=\mathrm{Ni}$.

\section{Magnetization-direction dependence of the spin response}

The direction of the magnetization vector $\boldsymbol{M}$ is often rotated in out-of-plane or in-plane directions in experiments [35,67]. The magnetization-direction unit vector $\mathcal{M}$ can be written as $\mathcal{M}=(\sin \theta \cos \phi, \sin \theta \sin \phi, \cos \theta)$, where $\phi$ and $\theta$ are the azimuthal and polar angles, respectively. To capture the $\mathcal{M}$ dependence of $\boldsymbol{\chi}^{S}$, we self-consistently compute $\chi^{S}$ at different $(\theta, \phi)$ values. We choose the $12 \mathrm{Pt} / 2 \mathrm{Co}$ system for these simulations and consider the ME spin response on the first Co atom at the interface with the Pt layer. In particular, we compute $\chi^{S}(\theta, \phi)$ for $\mathcal{M}$ constrained in the $x-y$ plane (Fig. 4) and $\mathcal{M}$ constrained in the $x-z$ plane (Fig. 5).

In Fig. 4, we show the computed angular dependence of the tensor components when the magnetization is in-plane and rotated from angle $\phi=0^{\circ}$ (i.e., parallel to $\boldsymbol{u}_{x}$ ) to $360^{\circ}$. The panels reveal that spin ME tensor is, to great precision, given by the following parametric form:

$$
\chi^{S}=\left(\begin{array}{ccc}
\alpha_{1} \sin 2 \phi & \alpha_{2}+\alpha_{3} \cos 2 \phi & \alpha_{4} \cos \phi \\
-\alpha_{2}-\alpha_{3} \cos 2 \phi & -\alpha_{1} \sin 2 \phi & \alpha_{4} \sin \phi \\
\alpha_{5} \cos \phi & \alpha_{5} \sin \phi & 0
\end{array}\right),
$$

where $\alpha_{i}(i=1,2, \ldots, 5)$ are constants in units of $10^{-3} \hbar \frac{\mathrm{nm}}{\mathrm{v}}$, whose values are $\alpha_{1}=12.54, \alpha_{2}=-20.83, \alpha_{3}=11.50$, $\alpha_{4}=77.18$, and $\alpha_{5}=-8.36$. Note that this functional form only gives the $\phi$ dependence, that is, $\theta$ is kept constant to $90^{\circ}$. The computed angle dependence illustrates that the tensor elements, and thus the resulting SOTs, obey a distinct angular symmetry. Terms whose angular dependency varies as $2 \phi$ are even under magnetization reversal, while those that vary as $\phi$ are odd.

Let us consider, for example, the case where $\boldsymbol{M}$ is along $\boldsymbol{u}_{x}$, that is $\phi=0$. If we apply a field $\boldsymbol{E}$ along $\boldsymbol{M}$, the only relevant nonzero elements, that is, the elements that give rise to a torque, are $\chi_{y x}^{S}$ and $\chi_{z x}^{S}$. The former tensor element is even in the magnetization, giving consistently a " $-\alpha_{2}-\alpha_{3} \cos 2 \phi$ " angular dependence, while the latter element is odd in $\boldsymbol{M}$, 


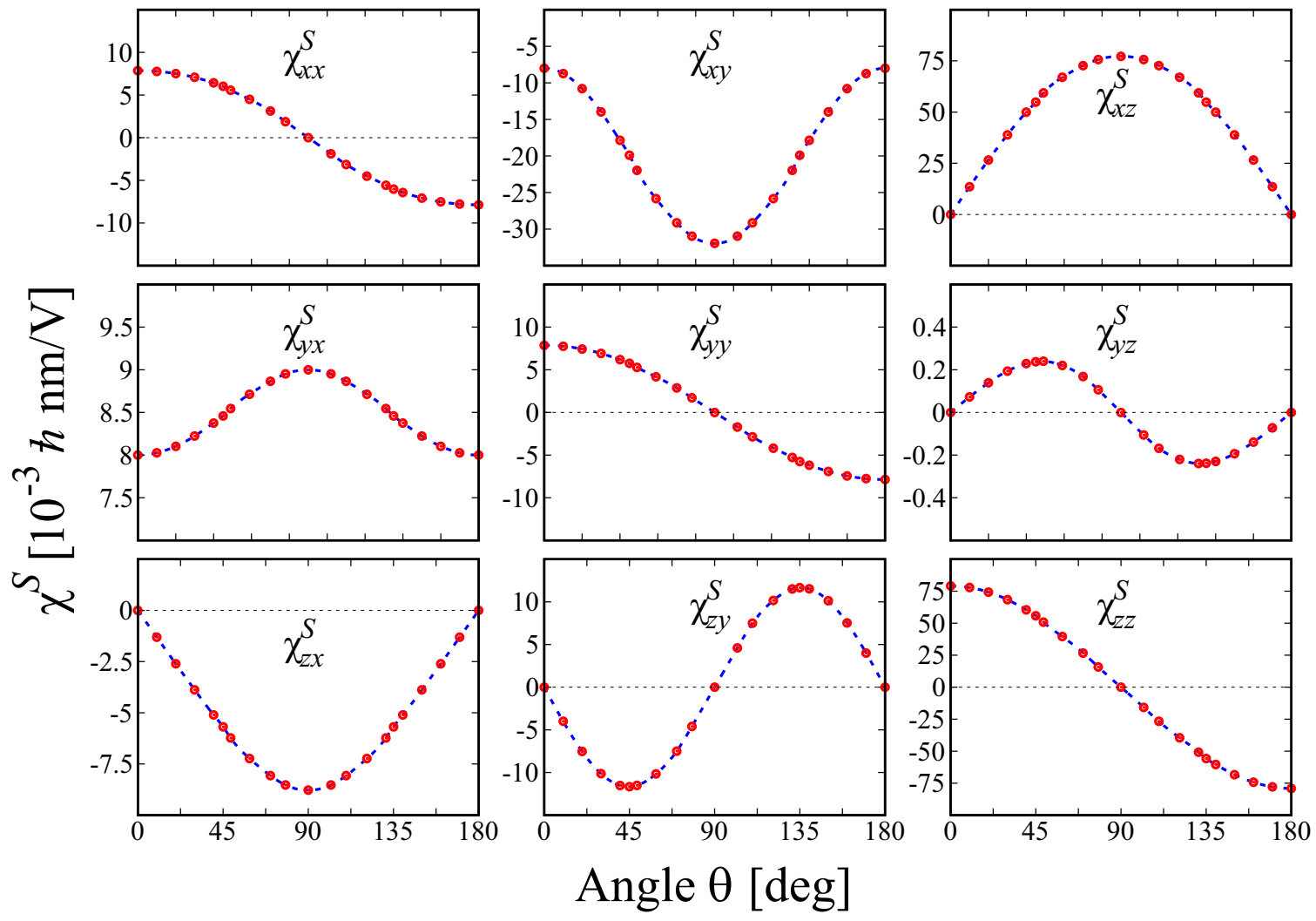

FIG. 5. Computed angular dependence of the spin ME tensor $\chi^{S}$ of the $12 \mathrm{Pt} / 2 \mathrm{Co}$ system at the first Co atom for magnetizations in the $x-z$ plane, given as a function of the polar angle $\theta$ (and for $\phi=0$ ).

giving a $\cos \phi$ dependence. Note that the expression (21) for the SOT contains the cross product $\boldsymbol{M} \times\left(\chi^{S} \boldsymbol{E}\right)$, which implies that an additional trigonometric function $(\sin \phi, \cos \phi)$ appears in the SOT.

Analogous calculations have been carried out for $\boldsymbol{M}$ being varied along the polar angle $\theta$, with $\phi$ set to 0 . Figure 5 shows the computed $\theta$-angle dependence of the ME tensor $\chi^{S}$ for the $12 \mathrm{Pt} / 2 \mathrm{Co}$ system. It can be recognized that the angular variation of the matrix elements obeys the $\theta$ dependence

$$
\chi^{S} \propto\left(\begin{array}{ccc}
\cos \theta & \beta_{1}+\cos 2 \theta & \cos \theta \\
\beta_{2}-\cos 2 \theta & \cos \theta & \sin 2 \theta \\
-\sin \theta & -\sin 2 \theta & \cos \theta
\end{array}\right)
$$

where $\beta_{i}$ and $\beta_{2}$ are constants. Here, we only give the generic trigonometric dependence of the tensor elements. In this case, there are nine constants needed to describe the angle dependence precisely as in Eq. (27).

The angular dependence of the $\chi^{S}$ tensor was computed for a particular atomic position in the bilayer. Performing further calculations, we find that the obtained angular dependence [Eqs. (27) and (28)] is valid at any atomic position in the layer, albeit with vanishing constants for the odd-in- $\boldsymbol{M}$ components when the atomic layer is close to the vacuum/Pt interface. For each atomic position there are different constants for the tensor components, however, a decisive property is, whether the tensor component is $\boldsymbol{E}$-transverse, $\boldsymbol{M}$-transverse, or $\boldsymbol{M}$-longitudinal. As illustrated in Table I, in this way equivalent tensor elements can be identified for different $\boldsymbol{M}$ directions.
In Fig. 6, we compare the atomic-layer resolved profiles of $\boldsymbol{\chi}^{S}$ for the $6 \mathrm{Pt} / 2 \mathrm{Ni}$ system, for $\boldsymbol{M} \| \boldsymbol{u}_{x}$ and $\boldsymbol{M} \| \boldsymbol{u}_{x}$. We compare the $\boldsymbol{E}_{\perp}, \boldsymbol{M}_{\perp}$, and $\boldsymbol{M}_{\|}$components, which allows us to track the physically equivalent quantities with respect to the SOT symmetry (FL or DL). We use in Fig. 6 the superscript $\boldsymbol{u}_{z}\left(\boldsymbol{u}_{x}\right)$ to denote quantities computed with $\boldsymbol{M} \| \boldsymbol{u}_{z}\left(\boldsymbol{M} \| \boldsymbol{u}_{x}\right)$. For most of the atomic positions, the corresponding tensor elements for both $\boldsymbol{M} \| \boldsymbol{u}_{z}$ and $\boldsymbol{M} \| \boldsymbol{u}_{x}$ show a strikingly similar behavior, with the exception of the $\chi_{x y}^{S}$ element that differs most in the Ni layer. The similar behavior and approximately identical values illustrate that the tensor elements transform in an almost rigid manner when the magnetization vector is rotated. The larger difference in the $\chi_{x y}^{S}$ component suggest that the orientation of $\boldsymbol{M}$ has a stronger influence on the $\mathrm{Pt} / \mathrm{Ni}$ interface electronic structure. Note that although $\chi_{x y}^{S, \boldsymbol{u}_{x}}$ and $\chi_{x y}^{S, \boldsymbol{u}_{z}}$ differ significantly at the Pt/Ni interface [see Fig. 6(a)], $\chi_{x y}^{S, \boldsymbol{u}_{x}}$ does not become effective, as it would give rise to an induced magnetization along the equilibrium magnetization, and can therefore not contribute to SOT.

For the $\boldsymbol{M}_{\perp}$ components, shown in Fig. 6(c), very similar magnitudes are observed but there is an opposite sign in the $\chi_{x x}^{S, \boldsymbol{u}_{z}}$ and $\chi_{z x}^{S, \boldsymbol{u}_{x}}$ components. This sign reversal is easily recovered using our proposed classification. Indeed, in the case $\boldsymbol{M} \| \boldsymbol{u}_{z}$, one finds from Eq. (16) for the corresponding component

$$
\chi_{x x}^{\boldsymbol{u}_{z}}: \delta \boldsymbol{S} \propto\left(\boldsymbol{u}_{x} \times \boldsymbol{u}_{z}\right) \times \boldsymbol{u}_{z}=-\boldsymbol{u}_{x},
$$

while for $\boldsymbol{M} \| \boldsymbol{u}_{x}$, we have

$$
\chi_{z x}^{\boldsymbol{u}_{x}}: \delta \boldsymbol{S} \propto\left(\boldsymbol{u}_{x} \times \boldsymbol{u}_{z}\right) \times \boldsymbol{u}_{x}=+\boldsymbol{u}_{z},
$$



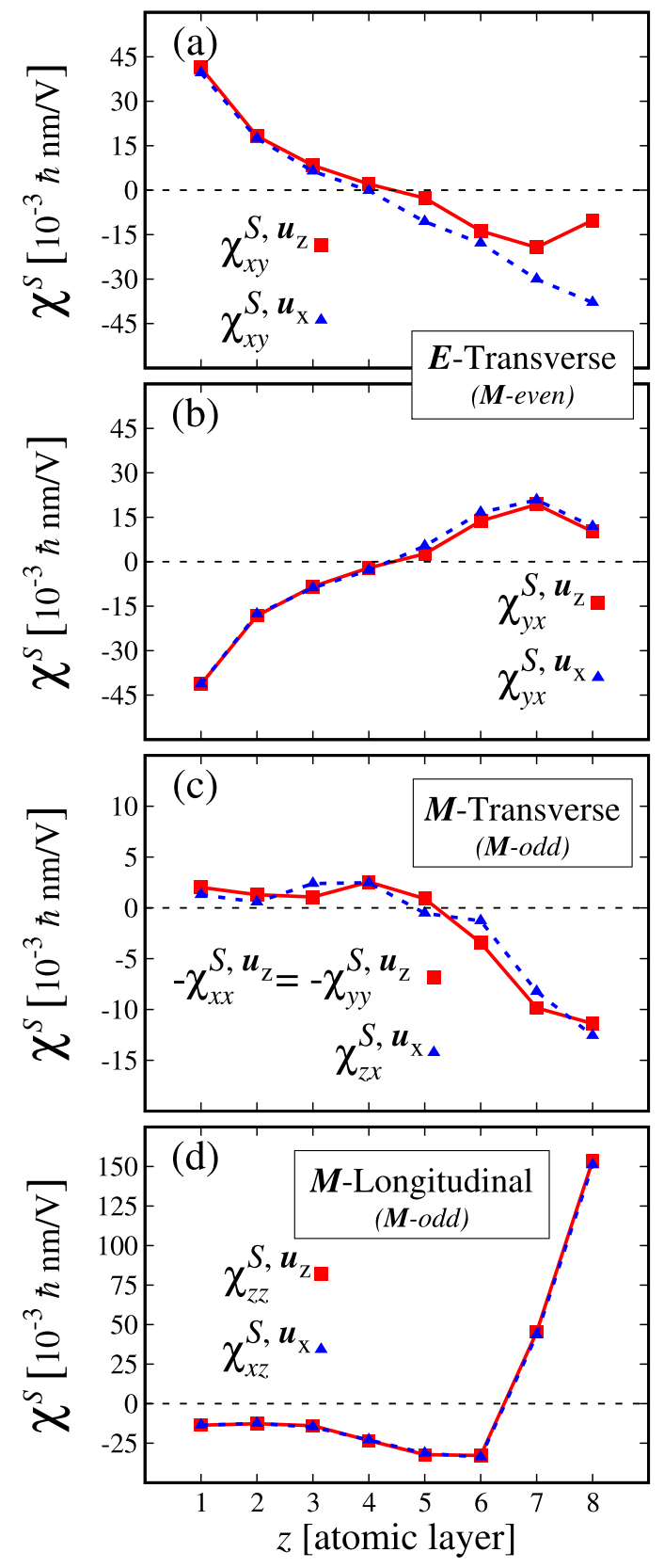

FIG. 6. Magnetization-direction dependence of the spin ME susceptibility tensor $\chi^{S}$ for $6 \mathrm{Pt} / 2 \mathrm{Ni}$. Calculated data are given for $\boldsymbol{M} \| \boldsymbol{u}_{z}\left(\boldsymbol{M} \| \boldsymbol{u}_{x}\right)$ by the red squares (blue triangles). When the magnetization direction switches from $\boldsymbol{u}_{z}$ to $\boldsymbol{u}_{x}$, the transverse components $\chi_{x y}^{S, \boldsymbol{u}_{z}}$ and $\chi_{y x}^{S, \boldsymbol{u}_{x}}$ are mapped onto themselves, with $\chi_{x y}^{S}$ being notably modified close to the $\mathrm{Pt} / \mathrm{Ni}$ interface while $\chi_{y x}^{S}$ is barely affected. The $\boldsymbol{M}$-transverse components $\chi_{x x / y y}^{S, \boldsymbol{u}_{z}}$ are mapped onto $-\chi_{z x}^{S, \boldsymbol{u}_{x}}$. The $\boldsymbol{M}$-longitudinal $\chi_{z z}^{s, u_{z}}$ component is transformed onto $\chi_{x z}^{S, u_{x}}$.

which perfectly captures the sign reversal. The $\boldsymbol{M}$-longitudinal $\chi^{S}$ components, lastly, follow the approximate transformation behavior quite well, see Fig. 6(d). These calculations illustrate the usefulness of the classification of the tensor elements according $\boldsymbol{E}_{\perp}, \boldsymbol{M}_{\perp}$, and $\boldsymbol{M}_{\|}$for identifying their resulting torque properties.

\section{Electronic lifetime dependence}

In the above calculations, we employed the intraband and interband electron lifetimes $\left(\hbar \tau_{\text {intra }}=0.220 \mathrm{eV}, \hbar \tau_{\text {inter }}=\right.$ $0.272 \mathrm{eV}$ ) obtained from fitting the calculated linear-response conductivity to experimental electrical conductivities of $\mathrm{Pt}$ films [51]. In general, these electronic lifetimes depend on the sample purity and microstructure. To investigate the influence of these lifetimes on the nonequilibrium spin and orbital polarizations we have varied these parameters in our calculations, adopting the $12 \mathrm{Pt} / 2 \mathrm{Co}$ and $14 \mathrm{Pt}$ films as typical model systems. We consider the case $\boldsymbol{M} \| \boldsymbol{u}_{z}$ for the $12 \mathrm{Pt} / 2 \mathrm{Co}$ film, for which the nonzero spin tensor elements are $\chi_{y x}^{S}, \chi_{x x}^{S}$, and $\chi_{z z}^{S}$. For the $14 \mathrm{Pt}$ film only the tensor element $\chi_{y x}^{S}$ is nonzero, cf. the results shown in Fig. 2. At this point it is relevant to mention that the $\boldsymbol{M}$-transverse tensor element $\chi_{x x}^{S}$ has only interband contributions, whereas the $\boldsymbol{E}$-transverse spin polarization $\chi_{y x}^{S}$ has both intra- and interband contributions. The calculated dependence of the torque-related, spin ME responses on the electronic broadening $\hbar \delta(=\hbar / \tau)$ is shown in Fig. 7. The intraband part of $\chi_{y x}^{S}$ increases linearly for decreasing lifetime broadening, as expected from Eq. (3). The interband contributions, conversely, vary with the lifetime broadening but become more broadening independent for large values of $\delta$. For small $\delta$ the interband contributions converge to nonzero values (but the values for $\chi_{y x}^{S}$ become small). For realistic lifetime values $(\hbar / \tau \sim 0.25 \mathrm{eV})$ the intraand interband induced spin polarizations have the same magnitude and need to be treated on equal footing. Note that semiclassical Boltzmann transport theory only captures the intraband spin ME component [68], an approximation that is viable for very pure crystals with long electron lifetimes.

In the limit of very small electronic broadenings, the intraband contribution to $\chi_{y x}^{S}$ will dominate completely the electrically induced spin polarization. This is the component that leads to a fieldlike SOT, see Table I. The intraband part of the spin response is commonly associated with the Rashba-Edelstein effect [53,60], consistent with the shape of the Rashba spin-orbit coupling Hamiltonian [25] that leads to a fieldlike torque in Landau-Lifshitz-Gilbert equations of spin dynamics. The spin Hall effect is conversely commonly associated with an interband contribution [24]. Such contribution is present both in $\chi_{y x}^{S}$ and $\chi_{x x}^{S}$, i.e., in the $E_{\perp}$ and $M_{\perp}$ terms. The SHE will thus contribute to both a fieldlike and a dampinglike SOT. The SHE is considered to lead to a DL torque [12] when using the Slonczewski model [1]. The SHE was however also proposed to lead to a FL torque [28,69], consistent with our calculations. The latter identification was made within the microelectronic circuit model, wherein the $\boldsymbol{M}_{\perp}\left(\boldsymbol{E}_{\perp}\right)$ component is mainly due to the SHE (SREE), when the spin mixing conductance is chiefly real $[28,69]$. To obtain such result, it is assumed that the transverse spin current and spin accumulation exists in the HM layer only, i.e., these quantities are zero in the FM layer. This might be a reasonable approximation for thicker layers, but in our case, where we compute atomistic quantities, we find a nonzero spin conductivity and spin accumulation (see Fig. 2) in the FM layer which is only two atoms thick. For a very small lifetime broadening the FL $\boldsymbol{E}_{\perp}$ contribution will dominantly stem from the intraband SREE and the DL $\boldsymbol{M}_{\perp}$ contribution from the 

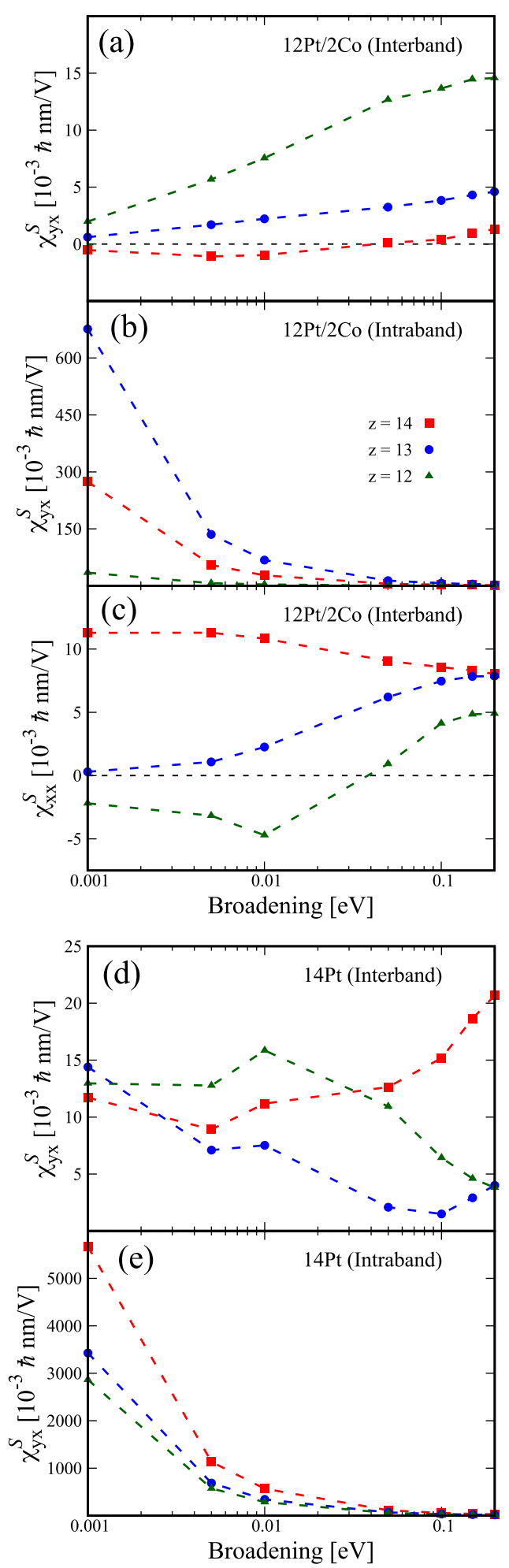

FIG. 7. Dependence of the interband and intraband contributions of the spin ME response $\chi^{S}$ on the electronic broadening $\delta$, computed for the $12 \mathrm{Pt} / 2 \mathrm{Co}$ and $14 \mathrm{Pt}$ systems. The $\chi_{x x}^{S}$ component is purely interband while $\chi_{x y}^{S}$ contains both interband and intraband contributions. The electronic broadening used in the main part of this article, $\delta=0.22-0.27 \mathrm{eV}$, is a realistic value for metallic systems.

SHE. We note however with respect to this discussion, that our DFT Hamiltonian contains the full form of the spin-orbit interaction and is thus different from the more elementary Bychkov-Rashba SOC [25], but it provides all materials' specific SOC effects.

For sake of completeness, we mention that the unusual $\boldsymbol{M}$ longitudinal ME susceptibilities and conductivities $\left(\chi_{z z}\right.$ and $\sigma_{z z}^{S_{z}}$ for $\boldsymbol{M} \| \boldsymbol{u}_{z}, \chi_{x z}$ and $\sigma_{x z}^{S_{x}}$ for $\left.\boldsymbol{M} \| \boldsymbol{u}_{x}\right)$ only have interband contributions.

\section{B. Orbital response}

\section{Atomic layer-resolved orbital response}

A similar analysis can be performed for the orbital response, both in terms of $\chi^{L}$ and $\sigma^{L}$. While similarities are observed, unique characteristic can be observed, too. To start with, we show in Fig. 8 the calculated layer-resolved orbital ME susceptibilities $\chi^{L}$ and orbital conductivities $\sigma^{L}$ for the $16 \mathrm{Pt} / 2 Y$ systems, for $\boldsymbol{M} \| \boldsymbol{u}_{z}$, similar to the spin counterparts shown in Fig. 2. For the sake of completeness, we provide in Appendix B analogous plots to Fig. 3 for the Pt-thickness dependence and to Figs. 4-6 for the angular dependence of the $\chi^{L}$ tensors and their transformation properties under rotation of the magnetization direction.

The layer-resolved results, shown in Fig. 8(a), reveal that, just like for the spin, the $\boldsymbol{E}$-transverse component in the pure $\mathrm{Pt}$ system resembles strongly the transport-induced accumulation of orbital angular momentum. The transverse conductivity $\sigma_{z x}^{L_{y}}$ in Fig. 8(d) is the orbital counterpart of the SHE, i.e., the OHE conductivity. Notwithstanding the analogy to the spin response, the overall shapes of $\chi_{y x}^{L}$ and $\sigma_{z x}^{L_{y}}$ show distinct features when compared to their spin counterparts. The shape of the $\chi_{y x}^{L}$ profile is considerably less smooth and the flat area of $\sigma_{z x}^{L_{y}}$ in the interior of the Pt layer is far more extended for all systems. In this area the orbital susceptibility, and thus the local accumulated orbital polarization, vanishes. Notably, considering the values obtained, we obtain a huge orbital response $\chi_{y x}^{L}$, roughly one order of magnitude larger than the spin counterpart. Also the OHE conductivity [Fig. 8(d)] is larger than the SHE conductivity. This finding is consistent with previous calculations of the OHE in bulk metals, which obtained an intrinsic OHE that is much larger than the SHE [42,43,70]. The huge induced $E_{\perp}$-orbital susceptibility is consistent with previous calculations for noncentrosymmetric antiferromagnets that obtained an OREE that was much larger than the SREE [46].

When it comes to the relative magnitude of the different configurations, $\boldsymbol{E}_{\perp}, \boldsymbol{M}_{\perp}$, and $\boldsymbol{M}_{\|}$, striking differences compared to the spin responses can be observed. Here, the orbital response at the interface is dominated by the $\boldsymbol{E}$-transverse component. We associate the $\boldsymbol{E}$-transverse $\chi_{y x}^{L}$ component, as before, to the orbital accumulation caused by both the OREE and the OHE. As we will see below, in particular the OREE (intraband) contribution to $\chi_{y x}^{L}$ is gigantic. The $\boldsymbol{M}$-transverse and $\boldsymbol{M}$-longitudinal orbital ME susceptibilities [Figs. 8(b) and 8(c)], are an order of magnitude smaller. Again, it is evident that the latter two orbital susceptibilities have a purely magnetic origin ( $\boldsymbol{M}$-odd) as they vanish for the nonmagnetic systems and are furthermore caused by the breaking of inversion symmetry. Similar to the case of the spin angular momentum, we identify the $\boldsymbol{M}$-transverse component 

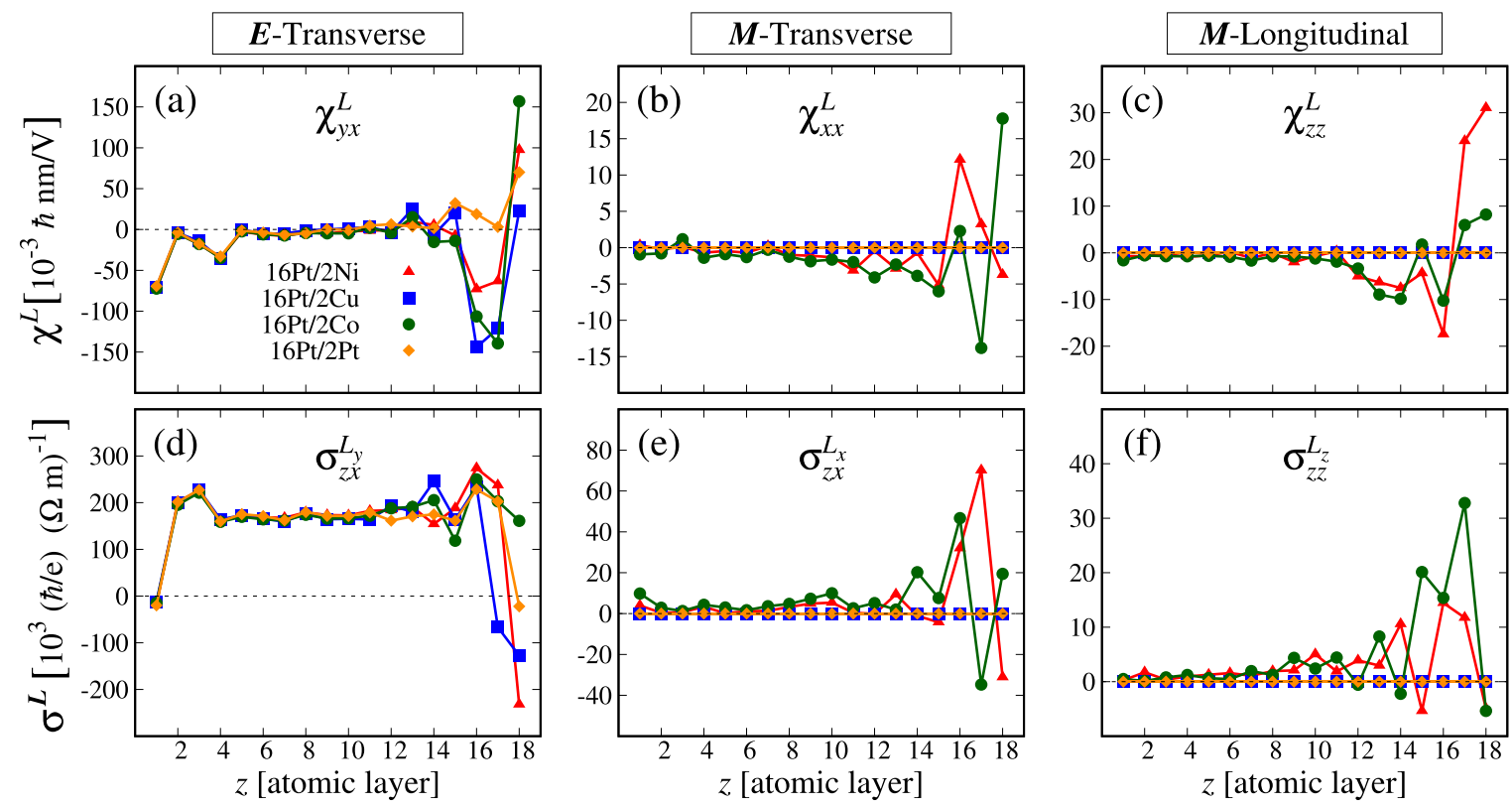

FIG. 8. Atomic layer-resolved nonzero components of the orbital ME susceptibility $\chi^{L}$ and the orbital conductivity $\sigma^{L}$ of the $16 \mathrm{Pt} / 2 Y$ films with $\boldsymbol{M} \| \boldsymbol{u}_{z}$. (a) The $\boldsymbol{E}$-transverse component $\chi_{y x}^{L}$, (b) $\boldsymbol{M}$-transverse component $\chi_{x x}^{L}$, and (c) the $\boldsymbol{M}$-longitudinal component $\chi_{z z}^{L}$. The corresponding components of the orbital conductivity tensor are given as (d) $\boldsymbol{E}$-transverse $\sigma_{z x}^{L_{y}}$, (e) $\boldsymbol{M}$-transverse $\sigma_{z x}^{L_{x}}$, and (f) $\boldsymbol{M}$-longitudinal $\sigma_{z z}^{L_{z}}$. The $\boldsymbol{E}$-transverse component $\sigma_{z x}^{L_{y}}$ is associated with the OHE conductivity, conventionally defined for bulk Pt. The $\boldsymbol{M}$-transverse components are nonzero only for the magnetic systems $(16 \mathrm{Pt} / 2 \mathrm{Ni}$ and $16 \mathrm{Pt} / 2 \mathrm{Co})$, and also the $\boldsymbol{M}$-longitudinal components arise from the spin polarization of the electronic states.

$\chi_{x x}^{L}$ therefore as being an orbital polarization due to the magnetic OHE.

A further significant difference between the spin and orbital ME susceptibilities is the rapid variation of the orbital ME susceptibilities in the last few layers of the $\mathrm{Pt} / Y$ interface. While the $\chi_{y x}^{S}$ component has positive values for the atomic monolayers in the vicinity of the interface [Fig. 2(a)], the orbital counterpart exhibits a sign change for the two topmost layers. A similar behavior can be observed for the $\boldsymbol{M}$-transverse components, $\chi_{x x}^{S}$ and $\chi_{x x}^{L}$. The unusual $\boldsymbol{M}$-longitudinal components exist, too, for the orbital ME susceptibility and conductivity, Figs. 8(c) and 8(f), but these quantities are, interestingly, much smaller than their spin counterparts.

The dependence of the orbital responses on the Pt-layer thickness is shown in Fig. 12 in Appendix A. Pt-layer thicknesses of about 8 monolayers provide stable values, for both the $\boldsymbol{E}_{\perp}$ and $\boldsymbol{M}_{\perp}$ components of the orbital ME susceptibilities.

Orbital transport and orbital polarization at interfaces are currently only poorly understood, and first measurements are being made [71-73] as well as theory developed [45,74]. Our calculations show that dependence of the orbital response $\chi^{L}$ on the magnetization direction exhibits similarities with the spin response $\chi^{S}$, as the nonzero components are the same for both cases. However, while the pair $\chi_{x y}^{S, \boldsymbol{u}_{z}} / \chi_{x y}^{S, \boldsymbol{u}_{x}}$ differs close to the Pt/Ni interface, we find that $\chi_{x y}^{L, \boldsymbol{u}_{z}} / \chi_{x y}^{L, \boldsymbol{u}_{x}}$ are virtually identical. This suggests a different, much smaller, dependence of orbital polarization on the magnetization direction at an interface.

Next, we have investigated the dependence of the induced orbital polarization on the magnetization direction. In the
Appendix B, in Figs. 13 and 14, we show the computed dependence of the components of $\chi^{L}$ on the magnetization angles $\phi$ and $\theta$, respectively. The $a b$ initio computed angle dependence of $\chi^{L}$ bears several similarities to the angle dependence of the spin susceptibility $\chi^{S}$, shown in Figs. 4 and 5. The components of the tensor $\chi^{L}$ follow a trigonometric dependence on the angles $\theta$ and $\phi$, but not identical to the ones given by Eqs. (27) and (28). However, for angles where a $\chi_{i j}^{S}$ component is zero, the corresponding $\chi_{i j}^{L}$ component is also zero. The symmetry of the $\chi^{L}$ and $\chi_{i j}^{S}$ tensor components with respect to $\boldsymbol{M}$, i.e., odd or even in $\boldsymbol{M}$, is also identical. A most significant difference is the dominance of the $\boldsymbol{E}$-transverse components $\chi_{x y}^{L} \approx-\chi_{y x}^{L}$ that are an order of magnitude larger than the other components.

Further insight is obtained from considering the dependence of the orbital ME susceptibilities on the electronic lifetime. To exemplify the broadening effect we consider the $12 \mathrm{Pt} / 2 \mathrm{Co}$ and $14 \mathrm{Pt}$ systems. In Fig. 9, we show the computed dependence of the nonzero orbital ME tensor elements on the lifetime broadening. Similar to the spin ME tensor elements, the intraband contributions increase linearly for decreasing broadening, whereas the interband contributions approach nonzero values (except for $\chi_{y x}^{L}$ ). The orbital ME susceptibilities reach however considerably larger values; for example, the intraband contribution to $\chi_{y x}^{L}$ is a factor of 150 larger than its spin counter part for the last Co atom of the $12 \mathrm{Pt} / 2 \mathrm{Co}$ system. This implies that in the limit of very pure crystals the $\boldsymbol{E}$-transverse interband $\chi_{y x}^{L}$ element, associated with the OREE, will dominate completely the response of this system. This conversely implies that the electrically induced orbital polarization has then always a pure Rashba symmetry, 

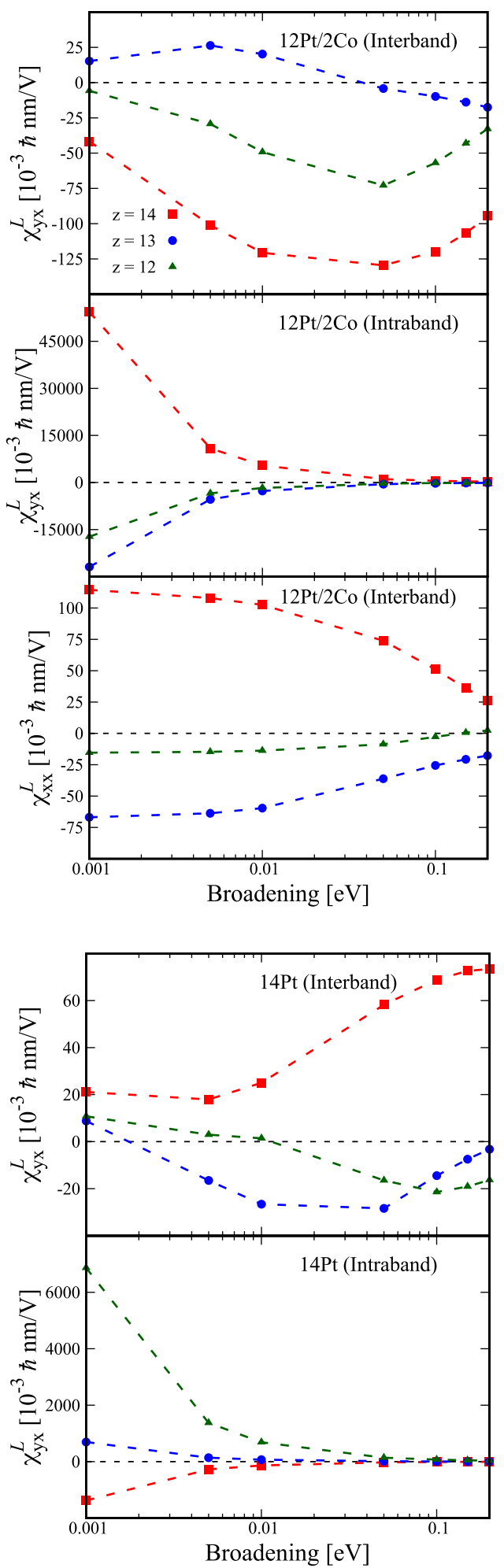

FIG. 9. Dependence of the interband and intraband contributions of the orbital ME response $\chi^{L}$ on the electronic broadening $\delta$ for the $12 \mathrm{Pt} / 2 \mathrm{Co}$ and $14 \mathrm{Pt}$ systems. Note that the orbital ME response is roughly one to two orders of magnitude larger than the spin ME response.

i.e., the induced orbital moment is perpendicular to the electric field direction. This is in accordance with previous calculations for noncentrosymmetric antiferromagnets that showed

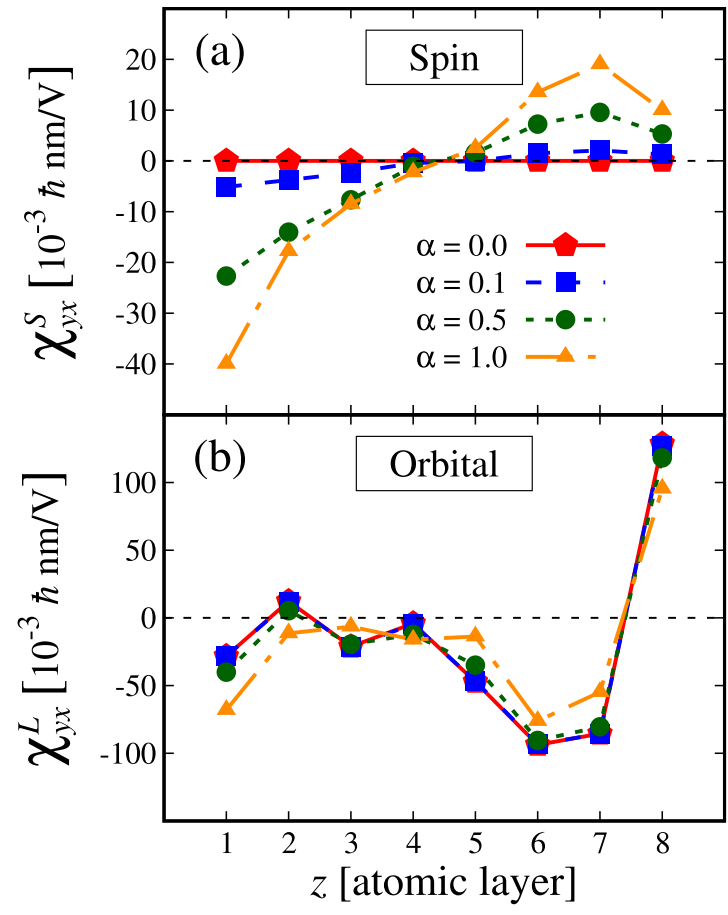

FIG. 10. Scaling behavior of (a) $\chi_{y x}^{S}$, and (b) $\chi_{y x}^{L}$ as a function of the SOC scaling parameter $\alpha$, calculated for the $6 \mathrm{Pt} / 2 \mathrm{Ni}$ system $\left(\boldsymbol{M} \| \boldsymbol{u}_{z}\right)$. The $\boldsymbol{E}_{\perp}$ component of the spin ME susceptibility (a) scales linearly with $\alpha$, and represents a SOC-induced quantity. The $\boldsymbol{E}_{\perp}$ component of the orbital ME susceptibility (b) exists even without SOC.

that the OREE has perfect Rashba symmetry and is considerably larger than the SREE that does not give a perfectly $\boldsymbol{E}$-orthogonal spin response [46].

\section{Dependence on spin-orbit coupling}

To investigate the dependence of the spin and orbital ME susceptibilities and conductivities we can vary the strength of the spin-orbit coupling in the calculations. To do this, we artificially introduce a SOC scaling parameter $\alpha$ in the DFT calculations such that $\hat{H}_{0}$ can be written as $\hat{H}_{0}=\hat{H}_{\mathrm{sc}}+\alpha \hat{H}_{\mathrm{soc}}$ where $\hat{H}_{\mathrm{sc}}$ is the scalar-relativistic part of the Hamiltonian and $\hat{H}_{\text {soc }}$ the SOC part. We find that without the $\hat{H}_{\text {soc }}$ term the whole spin ME susceptibility $\chi^{S}$ and spin conductivity $\sigma_{i j}^{S_{k}}$, with indices such that $\epsilon_{i j k}=0$, vanish. Obviously, as the electric field $\boldsymbol{E}$ couples only to the electron's position operator $\hat{\boldsymbol{r}}$, relevant to the electron's orbital motion, SOC is necessary to provide a coupling to the spin. Thus these spin quantities are completely induced by the SOC. For $\chi^{L}$, the story is quite different. When $\alpha$ is set to zero, $\chi_{x y}^{L}$ and $\chi_{x y}^{L}$, as well as $\sigma_{x z}^{L_{x}}$ and $\sigma_{y z}^{L_{x}}$, are nonzero and are actually not really affected by the modified SOC strength, a feature of the that has been noted before for the OHE conductivities $[42,43]$ and for the orbital polarizations induced by the OREE [46].

In Fig. 10, we show comprehensive results for the layerresolved profile of $\chi_{x y}^{S}$ and $\chi_{x y}^{L}$ for $6 \mathrm{Pt} / 2 \mathrm{Ni}$, computed for $\alpha=0,0.1,0.5$, and 1 , with $\alpha=1$ corresponding to the intrinsic SOC strength. It is evident from Fig. 10(a) that spin ME susceptibility is a pure SOC effect that scales linearly 
with the SOC. The situation is different for the orbital ME susceptibility, which exhibits practically no dependence on the SOC strength, see Fig. 10(b). Clearly, the induced $\boldsymbol{E}$ transverse orbital polarization represents the nonrelativistic response of the electron system to the electric field. That the induced orbital moment is nonrelativistic and perpendicular to the electric field $\boldsymbol{E}$ can be recognized from the one-electron operator expression

$$
\frac{d}{d t}(\delta \hat{\boldsymbol{L}})=\hat{\boldsymbol{r}} \times e \boldsymbol{E},
$$

that does not require SOC. For all other spin and orbital susceptibility components, as well as for the spin conductivity tensors elements, we find that these scale linearly with the size of the SOC, i.e., these are quantities induced by the SOC.

\section{DISCUSSION}

\section{A. Spin-orbit torque}

Freimuth et al. [75] evaluated directly the SOT using a different approach to the perturbative DFT framework. While our computational method is distinct from theirs, we can evaluate the SOT $\mathcal{T}$ in a similar fashion. Using Eq. (21), we can write

$$
\delta \boldsymbol{B} \approx \underbrace{\frac{\left\langle V_{\mathrm{KS}}^{\downarrow}-V_{\mathrm{KS}}^{\uparrow}\right\rangle}{2 \mu_{B}|\boldsymbol{S}|} \chi^{S}}_{\boldsymbol{\chi}_{\mathrm{SOT}}} \boldsymbol{E},
$$

where $V_{\mathrm{KS}}^{\downarrow}\left(V_{\mathrm{KS}}^{\uparrow}\right)$ is the Kohn-Sham effective potential for minority (majority) spin electrons and $S$ the equilibrium spin angular momentum. As mentioned in Sec. II D 2, this is an approximation of $\delta \boldsymbol{B}$.

We define furthermore $\chi_{\text {SOT }}$ as the SOT spin susceptibility tensor in units of $\mathrm{TmV}^{-1}$. Since our computational approach involves quantities evaluated for each atomic site, we can access a layer-resolved $\boldsymbol{B}_{\text {SOT }}$.

For the thickest magnetic systems, 16Pt/2Co and $16 \mathrm{Pt} / 2 \mathrm{Ni}$, we find that the $\boldsymbol{E}_{\perp}$ contribution to the SOT at the first (second) layer of $\mathrm{Ni}$ is $0.0032(0.0020) \mathrm{mT} \mathrm{cm} \mathrm{V}^{-1}$ and $0.0019(0.0007) \mathrm{mT} \mathrm{cm} \mathrm{V}^{-1}$ for Co. For the $\boldsymbol{M}_{\perp}$ contribution, we find $0.0020(0.0030) \mathrm{mT} \mathrm{cm} \mathrm{V}^{-1}$ for the first (second) layer of $\mathrm{Ni}$ and $0.0019(0.0020) \mathrm{mT} \mathrm{cm} \mathrm{V}^{-1}$ the first (second) layer of Co. These values are smaller than, but consistent with, those obtained by Freimuth et al. [27], because they used a much smaller broadening of electronic states.

The possible generation of large orbital torques has recently drawn attention [45]. However, although the orbital ME susceptibility is large, this does not automatically imply a large torque, because the induced orbital polarization can only couple to the static magnetic spin moment $\boldsymbol{M}$ via SOC. More precisely, in the commonly used formulation and implementation of DFT, the effective Kohn-Sham potential is not a functional of the orbital character, i.e., the exchangecorrelation field only couples to spin angular momentum. The influence of the $\chi^{L}$ on the SOT is nonetheless included in our calculations as the induced $\delta \boldsymbol{L}$ couples to $S$ via SOC (and vice versa). Notwithstanding, theoretical efforts have recently been devoted to predicting the orbital torque $[45,76]$ and experimental efforts are being undertaken to detecting the
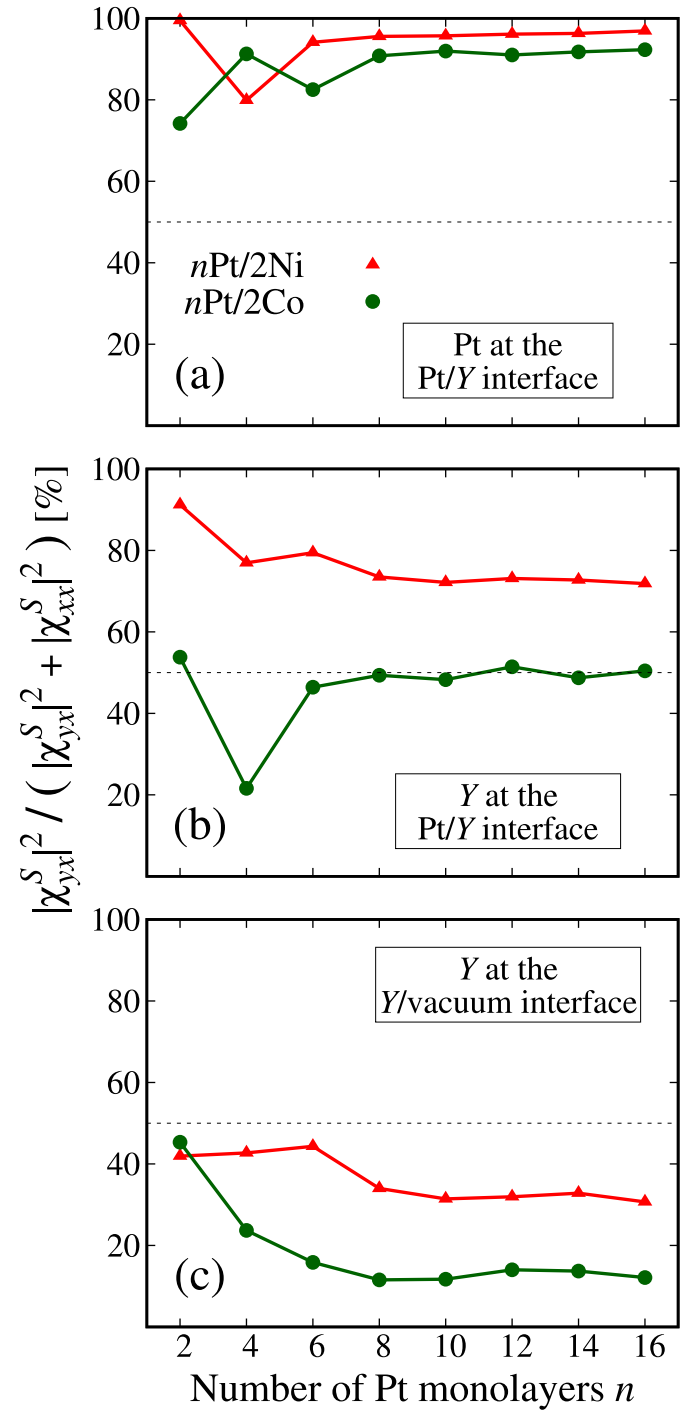

FIG. 11. Computed ratio of the fieldlike and (vectorial summed) total SOT as function of $\mathrm{Pt}$ layer thickness for the $n \mathrm{Pt} / 2 \mathrm{Co}$ and $n \mathrm{Pt} / 2 \mathrm{Ni}$ systems with $\boldsymbol{M} \| \boldsymbol{u}_{z}$. The fieldlike $\boldsymbol{E}$-transverse torque is proportional to $\chi_{y x}^{S}$ and the dampinglike torque to $\chi_{x x}^{S}$. (a) The ratio at the $\mathrm{Pt}$ atom at the $\mathrm{Pt} / Y$ interface, (b) the ratio at the $Y$ atom at the $\mathrm{Pt} / Y$ interface, and (c) at the $Y$ atom at the $Y$ /vacuum interface $(Y=\mathrm{Co}$ or $\mathrm{Ni})$.

orbital polarization and torque and disentangling it from the spin torque $[72,73,77,78]$.

\section{B. Relative sizes of fieldlike and dampinglike torques}

The layer-resolved SOTs are dominantly defined by the size of the spin ME susceptibility $\chi^{S}$. Any resulting torque can be decomposed, as customary is done, in an even-in- $\boldsymbol{M}$ FL component and and odd-in- $\boldsymbol{M}$ DL component. The linear dependence of the effective SOT magnetic field on $\chi^{S}$ permits to compare the relative sizes of the FL and DL SOTs. Considering the case $\boldsymbol{M} \| \boldsymbol{u}_{z}$, the $\boldsymbol{E}_{\perp}$ component $\chi_{y x}^{S}$ leads to the FL torque, while the $\boldsymbol{M}_{\perp}$ component $\chi_{x x}^{S}$ corresponds to the DL torque. We can then quantify the relative importance of the 

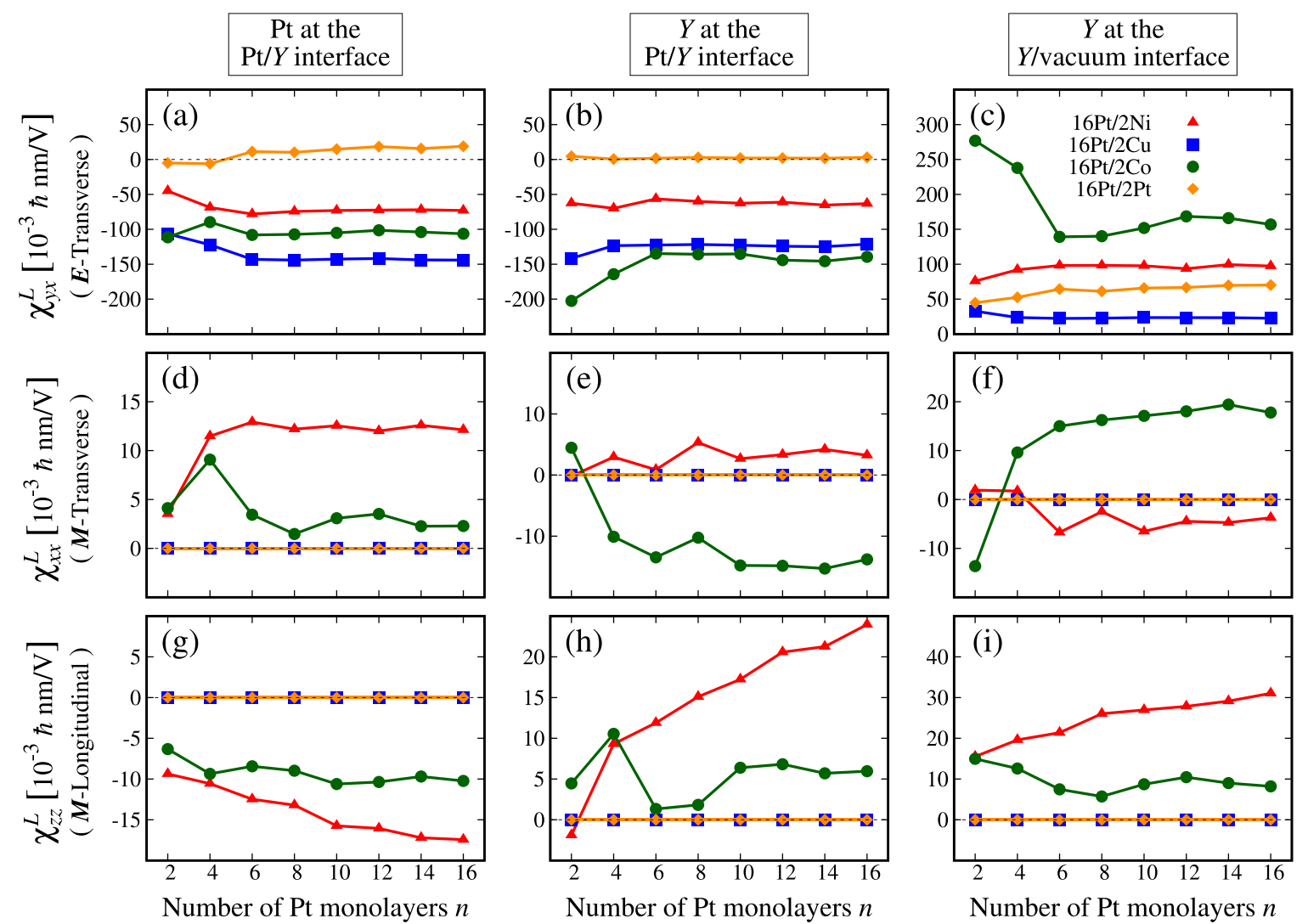

FIG. 12. Dependence of the orbital susceptibility tensor $\chi^{L}$ on the number of Pt monolayers $n$. (Top row) $\boldsymbol{E}$-transverse component of $\chi^{L}$ for (a) the $\mathrm{Pt}$ atom at the $\mathrm{Pt} / Y$ interface, (b) the $Y$ atom at the $\mathrm{Pt} / Y$ interface, and (c) the $Y$ atom at the $Y /$ vacuum interface. Middle row: $\boldsymbol{M}$-transverse component of $\chi^{L}$ for (d) the $\mathrm{Pt}$ atom at the $\mathrm{Pt} / Y$ interface, (e) the $Y$ atom at the $\mathrm{Pt} / Y$ interface, and (f) the $Y$ atom at the $Y /$ vacuum interface. (Bottom row) $M$-longitudinal component of $\chi^{L}$ for (g) the $\mathrm{Pt}$ atom at the $\mathrm{Pt} / Y$ interface, (h) the $Y$ atom at the $\mathrm{Pt} / Y$ interface, and (i) the $Y$ atom at the $Y$ /vacuum interface.

two torques by computing the ratio

$$
\frac{\left|\chi_{x y}^{S}\right|^{2}}{\left|\chi_{x y}^{S}\right|^{2}+\left|\chi_{x x}^{S}\right|^{2}} \times 100 \% \text {. }
$$

A value of $>50 \%(<50 \%)$ would then refer to a fieldlike (dampinglike) dominated torque. The square exponent accounts for the fact that we are comparing vectorial quantities. Note that $\left|\chi_{x x}^{S}\right|$ should be replaced by $\left|\chi_{z x}^{S}\right|$ for $\boldsymbol{M} \| \boldsymbol{u}_{x}$.

The calculated Pt-thickness dependence of this ratio for the $n \mathrm{Pt} / 2 \mathrm{Ni}$ and $n \mathrm{Pt} / 2 \mathrm{Co}$ systems is displayed in Fig. 11, for the last $\mathrm{Pt}$ monoatomic layer at the $\mathrm{Pt} / Y$ interface, as well as for the $Y$ monolayer at the Pt interface and at the vacuum interface. It can be observed that there is virtually no change for the computed torque ratio for Pt layer thicknesses beyond eight $\mathrm{Pt}$ monolayers. For the $\mathrm{Pt}$ monolayer at the $\mathrm{Pt} / Y$ interface, the induced torque is to $90 \%$ composed of the FL torque component, see Fig. 11(a). For the $Y$ monolayer at the $\mathrm{Pt} / Y$ interface, the torque consists for $\sim 75 \%$ of the FL component for $Y=\mathrm{Ni}$ and $\sim 50 \%$ for $Y=$ Co [Fig. 11(b)]. For the $Y$ monolayer at the $Y$ /vacuum interface, the torque consist for $\sim 30 \%$ of the FL component for $Y=\mathrm{Ni}$ and $\sim 10 \%$ for $Y=\mathrm{Co}$ [Fig. 11(c)]. This suggests that the $\mathrm{Pt} / \mathrm{Ni}$ interface is more transparent to spin currents from the Pt than the $\mathrm{Pt} / \mathrm{Co}$ interface, consistent with the better matching electronic structures of isoelectronic fcc Ni and Pt.
The torques resulting from the induced spin polarization on the two ferromagnetic $Y$ monolayers will be the most important ones for the magnetization switching. The torque on the ferromagnetic layer at the vacuum interface is thus approximately both field- and dampinglike, whereas the torque at the ferromagnetic layer adjacent to the $\mathrm{Pt}$ layer has a larger FL contribution. As the relative contribution of the $\boldsymbol{E}_{\perp}$ and $\boldsymbol{M}_{\perp}$ components differs in both $3 d$ monolayers, the direction of the total torque per monolayer will be different for each of the two $Y$ monolayers. The calculated atomic-layer specific torques are ideally suited to investigate currentdriven magnetization switching dynamics using atom-specific Landau-Lifshitz-Gilbert spin-dynamics simulations (see, e.g., Refs. [79-81]). Such simulations would provide layer-specific insight in how the magnetization of the ferromagnetic layers reverses under an applied electric field.

\section{CONCLUSIONS}

We have employed first-principles calculations to investigate the electric-field induced spin and orbital magnetoelectric susceptibility and the spin and orbital conductivity of heavy-metal $/ 3 d$-metal bilayer structures. For each orientation of the $3 d$ magnetization and the applied electric field we have shown that the susceptibility tensor and its associated conductivity tensor can be uniquely decomposed in components 


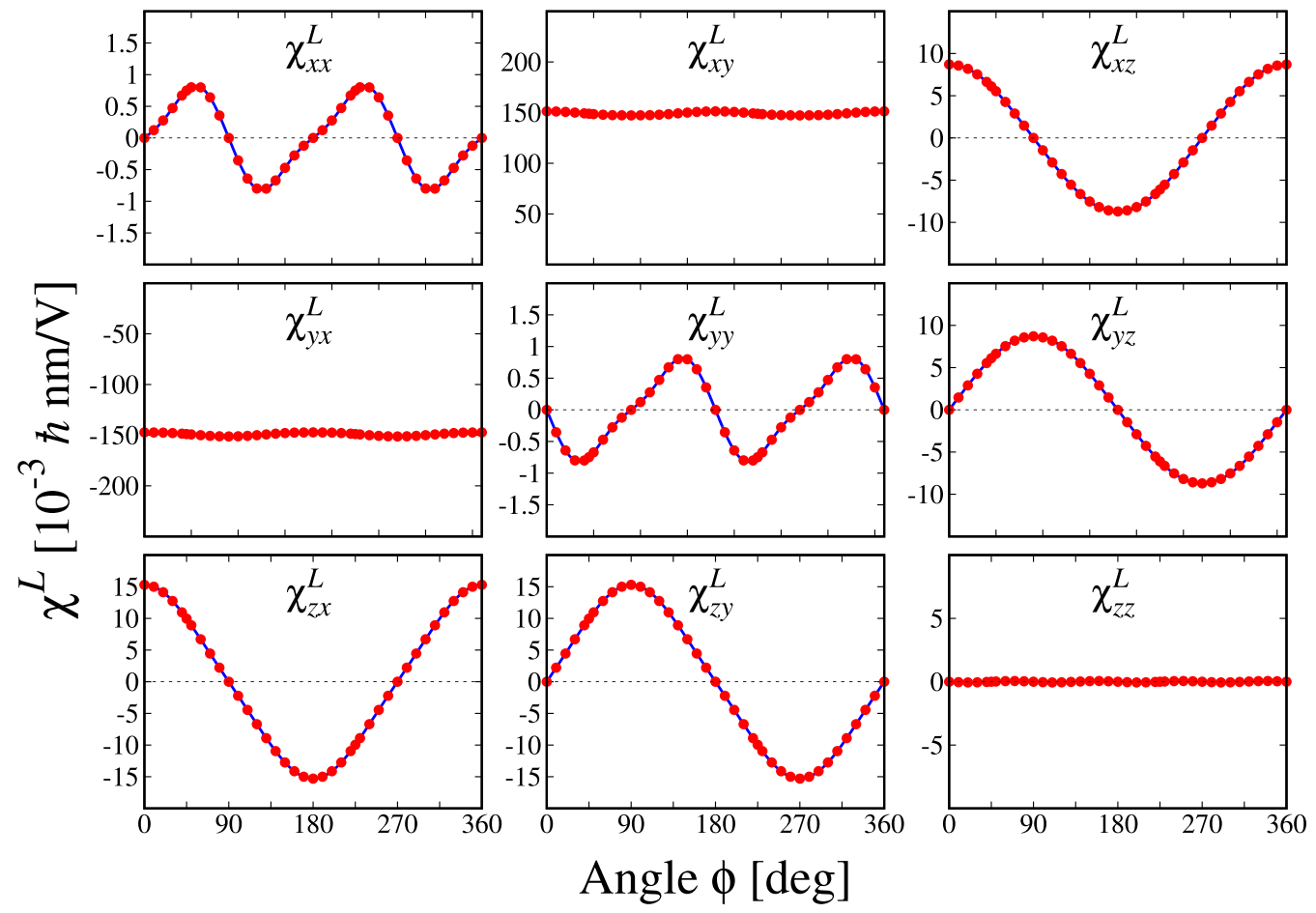

FIG. 13. Computed angular dependence of the orbital susceptibility tensor $\chi^{L}$ of the $12 \mathrm{Pt} / 2 \mathrm{Co}$ system at the interfacial Co atom for in-plane magnetizations, given as a function of the azimuthal angle $\phi$.

depending on the spatial symmetries, i.e., transverse electric $\boldsymbol{E}_{\perp}$, transverse magnetic $\boldsymbol{M}_{\perp}$, and longitudinal magnetic components $\boldsymbol{M}_{\|}$, as well as the magnetic symmetries (odd-in- $\boldsymbol{M}$ and even-in- $\boldsymbol{M}$, respectively). Our atomic-layer specific calculations of the tensors show that all components are highly dependent on the position of the atomic layer in the considered heterostructure.

Analyzing the properties of the computed ME susceptibilities, we have identified the even-in- $\boldsymbol{M}, \boldsymbol{E}_{\perp}$ components of $\chi^{S}$ as spin accumulation associated with both the SHE

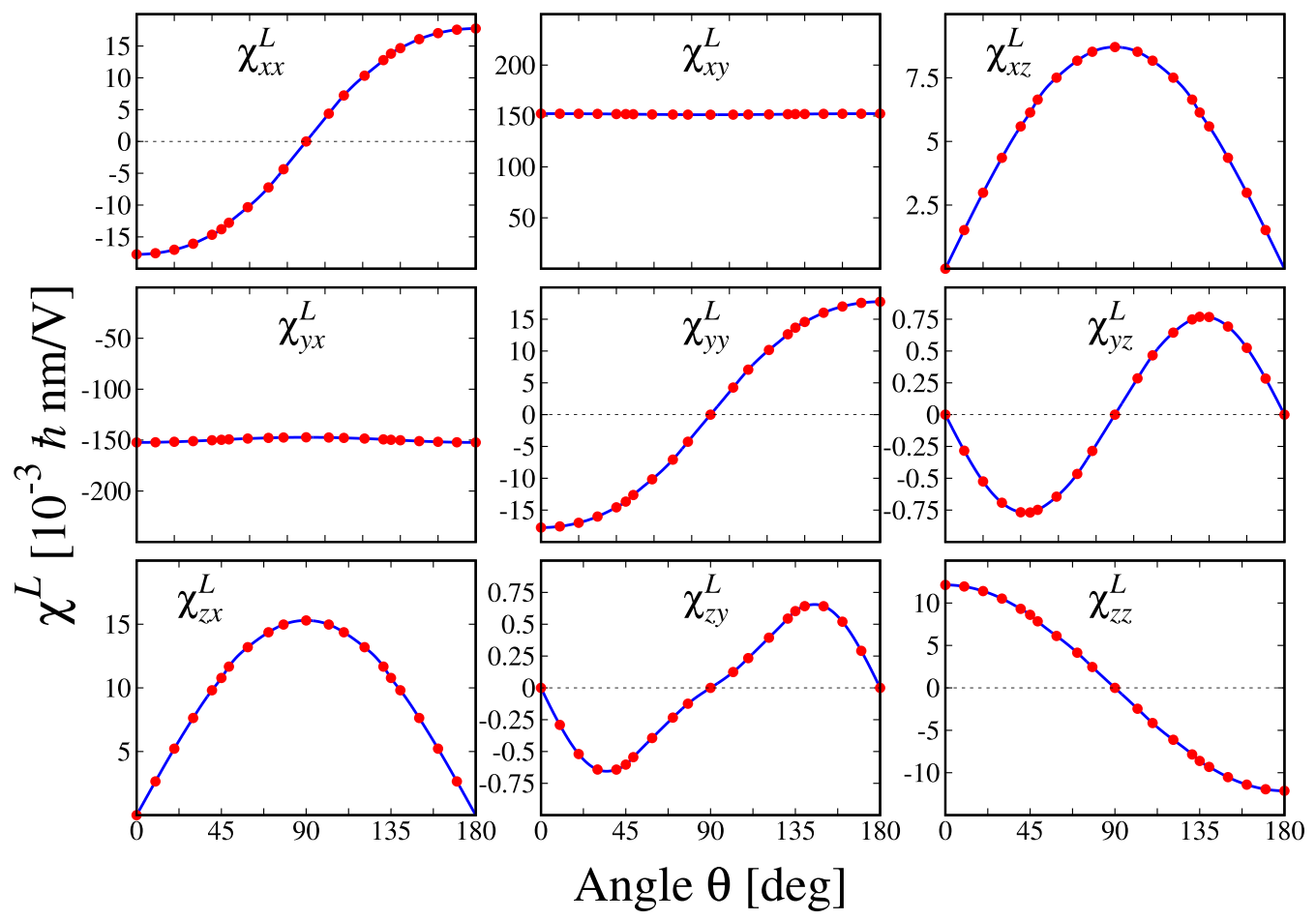

FIG. 14. Computed angular dependence of the orbital susceptibility tensor $\chi^{L}$ of the $12 \mathrm{Pt} / 2 \mathrm{Co}$ system at the interfacial Co atom for magnetizations in the $x-z$ plane, given as a function of the polar angle $\theta$. 
and SREE, and the odd-in- $\boldsymbol{M}, \boldsymbol{M}_{\perp}$ components with the magnetic SHE. We note however that our ab initio formulation uses a more general form of the SOC than the often used more elementary Rashba SOC. Extending the calculations to field-induced orbital polarizations, we have performed a similar analysis and decomposition for the orbital susceptibility tensor $\chi^{L}$ and orbital conductivity, $\sigma^{L}$. We have analyzed the relative importance of the different spin and orbital contributions as a function of Pt thickness. Both out-of-equilibrium $\boldsymbol{E}_{\perp}$ and $\boldsymbol{M}_{\perp}$ spin responses lead to atomic-layer dependent SOTs that are of the same order of magnitude, but act in perpendicular directions. We find that the $\boldsymbol{E}$-transverse spin accumulation is largest for the Pt layer at the $\mathrm{Pt} / 3 d$-metal interface. The $\boldsymbol{M}$-transverse spin accumulation, conversely, is larger at the $3 d$-vacuum interface. Our calculations show that both effects should be considered together when analyzing current-induced spin polarization in heavy-metal/ferromagnetic bilayer systems.

This perception is valid for electronic relaxation times that are realistic for metallic systems $(\hbar / \tau \approx 0.25 \mathrm{eV})$. For extremely pure materials, however, the intraband contribution to the $\boldsymbol{E}$-transverse induced spin polarization will be much larger than other (interband) contributions, leading to a predominant fieldlike SOT.

Considering the electric-field induced orbital polarization, we find that the $\boldsymbol{E}$-transverse orbital susceptibility and conductivity components are always much larger $(\sim 10 \times)$ than their $\boldsymbol{M}$-transverse orbital counterparts. In contrast to the spin counterparts, the $\boldsymbol{E}_{\perp}$-orbital ME susceptibility and OHE conductivity do not dependent on SOC. This exemplifies that the induced $\boldsymbol{E}$-transverse orbital polarization is the primary response of the electron system to the electric field and that the other, both spin and orbital, induced polarizations are generated from the nonzero $\boldsymbol{E}_{\perp}$-orbital susceptibility by SOC. The nonrelativistic orbital ME susceptibilities are ten to a hundred times larger than the corresponding relativistic spin susceptibilities. However, although the electrically induced orbital polarization is huge, it can only couple to the equilibrium spin moment via SOC.

The computed induced spin and orbital polarizations follow trigonometric functional dependencies on the magnetization direction angles that are consistent with the $\boldsymbol{M}$ symmetry of the ME susceptibility components. Of particular interest are the large $\boldsymbol{E}_{\perp}$-orbital ME components that are practically angle independent and antisymmetric, i.e., $\chi_{x y}^{L}=-\chi_{y x}^{L}$. The induced $\boldsymbol{E}$-transverse orbital polarization exhibits consequently a pure Rashba symmetry.

Our calculations show furthermore that there exists as well an electric-field induced spin and orbital polarization along the magnetization direction. This unusual spin-orbit effect does not exert a torque on the static magnetization. We propose that it could be possible to observe this $\boldsymbol{M}$-longitudinal effect in sensitive magneto-optical Kerr effect measurements (cf. Ref. [51]). Steps in this direction were recently taken for $\mathrm{Pt} / \mathrm{Co}$ thin films for which a change of the Co $\boldsymbol{M}_{\|}$moment was detected $[82,83]$.

When the magnetization direction changes, the spin and orbital responses also change. We have shown that the magnetization direction does have a strong influence on the spin and orbital responses, but that it is possible to track the evo-
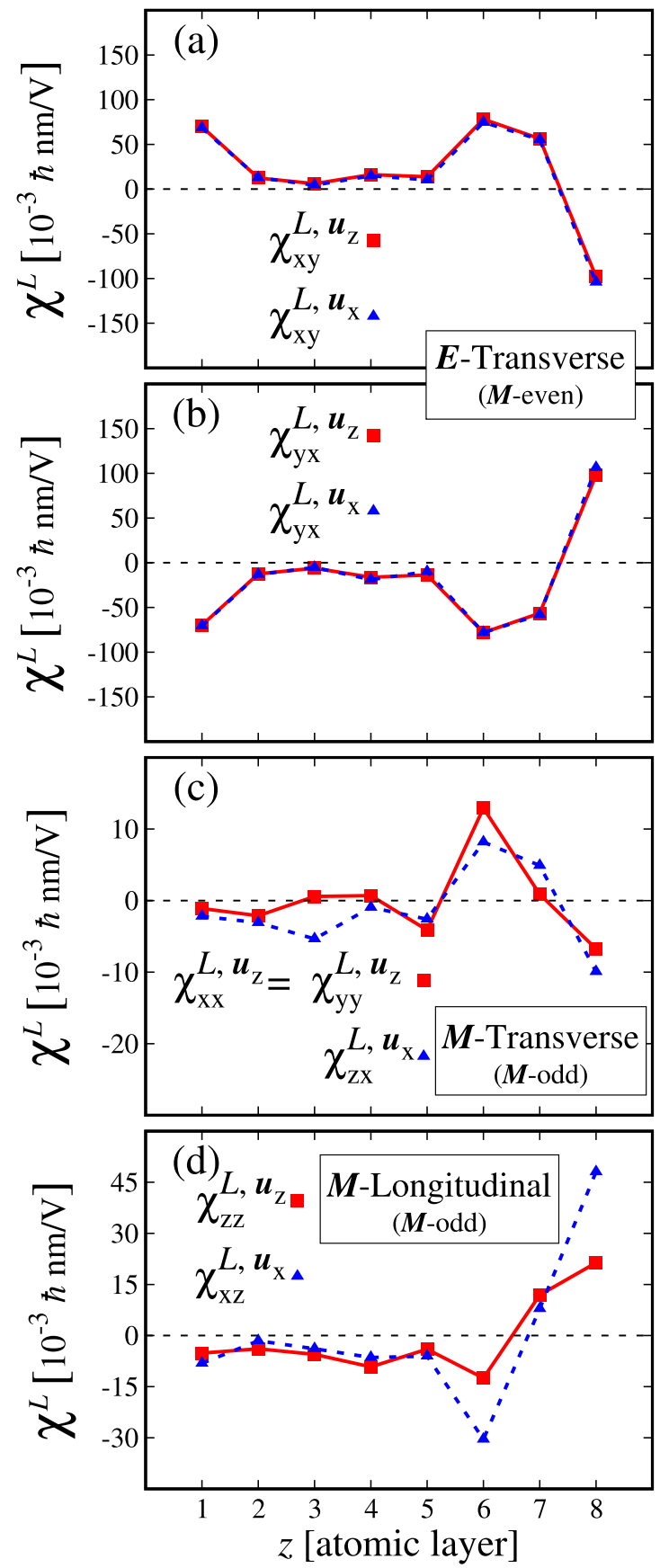

FIG. 15. Magnetization direction dependence of the orbital susceptibility tensor $\chi^{L}$ for $6 \mathrm{Pt} / 2 \mathrm{Ni}$. The data are shown for $\boldsymbol{M} \| \boldsymbol{u}_{z}$ ( $\boldsymbol{M} \| \boldsymbol{u}_{x}$ ) with the red squares (blue triangles). When the magnetization direction switches from $\boldsymbol{u}_{z}$ to $\boldsymbol{u}_{x}$, the transverse components $S_{x y}^{L, \boldsymbol{u}_{z}}$ and $S_{y x}^{L, \boldsymbol{u}_{x}}$ are mapped onto themselves. The $\boldsymbol{M}$-transverse components $\chi_{x x / y y}^{L, \boldsymbol{u}_{z}}$ are mapped onto $\chi_{z x}^{L, \boldsymbol{u}_{x}}$. The $\boldsymbol{M}$-longitudinal component $\chi_{z z}^{L, \boldsymbol{u}_{x}}$ is mapped onto $\chi_{x z}^{L, \boldsymbol{u}_{x}}$. The mapping of the components upon magnetization rotation respects our symmetry analysis, in terms of $\boldsymbol{E}_{\perp}, \boldsymbol{M}_{\perp}$, and $\boldsymbol{M}_{\|}$principal components.

lution of the individual components using simple, but robust, symmetry relations. This should aid the future investigation of SOT magnetization switching using atom-specific LandauLifshitz-Gilbert spin-dynamics simulations. 


\section{ACKNOWLEDGMENTS}

We thank the anonymous reviewer for constructive comments. This work has been supported by the Swedish Research Council (VR), the K. and A. Wallenberg Foundation (Grant No. 2015.0060), and the Swedish National Infrastructure for Computing (SNIC) (Grant No. 2018-05973). This work has been funded by the European Union's Horizon2020 Research and Innovation Programme under FET-OPEN Grant agreement No. 863155 (s-Nebula). The calculations were performed at the PDC Center for High Performance Computing and the Uppsala Multidisciplinary Center for Advanced Computational Science (UPPMAX).

\section{APPENDIX A: COMPUTATIONAL DETAILS}

As mentioned in Sec. II C, the calculations are performed in three steps. First, the structures are fully relaxed with the DFT package SIESTA [52]. The cell parameters and atomic positions of the pure $\mathrm{Pt}$ films are relaxed until the pressure reaches values below $0.001 \mathrm{GPa}$ and atomic forces on each atom are below $0.01 \mathrm{eV} / \AA$. Then, the cell parameter is fixed and two monolayers of $3 d$ elements $(\mathrm{Ni}, \mathrm{Co}$, or $\mathrm{Cu})$ are added. The atomic positions are then relaxed using the same criterion as before. All SIESTA calculations are performed using a $15 \times 15 \times 1$ Monkhorst-Pack grid [84] with an electronic temperature of $300 \mathrm{~K}$. The double $\zeta$ with polarization pseudoatomic basis set functions are used. The mesh-cutoff for real space integration is set to $250 \mathrm{Ry}$ and we use the generalized gradient approximation (GGA) for the exchange-correlation functional in the PBEsol parametrization [85]. All structures contain $20 \AA$ of vacuum to avoid spurious interactions with neighboring simulation cells.

Second, once the structures are relaxed, the ground-state Kohn-Sham wave functions and energies are computed using the accurate full-potential, all-electron code WIEN2K [49], with spin-orbit interaction included [86]. The product between the smallest muffin-tin radius $R_{M T}$ and the largest reciprocal vector $K_{\max }$ is set to $R_{M T} \times K_{\max }=8.5$ and the self-consistent spin-polarized density is computed using a $30 \times 30 \times 1 \mathrm{k}$ point Monkhorst-Pack grid. The computed spin moments for the $16 \mathrm{Pt} / 2 \mathrm{Ni}$ bilayer are $0.855 \mu_{B}$ and $0.760 \mu_{B}$ at Ni18 and Ni17, respectively. The spin moment on the Pt interface layer (Pt16) is $0.212 \mu_{B}$. For the $16 \mathrm{Pt} / 2 \mathrm{Co}$ bilayer the equivalent moments are $2.02 \mu_{B}, 1.942 \mu_{B}$, and $0.251 \mu_{B}$. The proximity induced moments in the Pt layer vanish within four layers.

Finally, the atom-resolved spin response tensors are then computed with a denser $200 \times 200 \times 1 k$ mesh. As the WIEN2K code uses atom-centered wave functions, we exploit here this property to compute them in an atom-projected fashion. The simulation cell is divided into two subspaces: muffin-tin spheres around each atom, in which the wave function is expanded in terms of spherical harmonics, and the interstitial region in which the wavefunction is given in terms of plane waves, i.e.,

$$
\Psi(\boldsymbol{r})=\sum_{\alpha} \Psi_{\alpha}\left(\boldsymbol{r}-\boldsymbol{R}_{\alpha}\right)+\Psi_{I}(\boldsymbol{r}),
$$

where the first right-hand term is the wave function about atom $\alpha$ and $\Psi_{I}(\boldsymbol{r})$ is the wave function in the interstitial. The atom-projected expected value of an operator $\hat{O}$ is taken as

$$
O_{\alpha}=\int d \boldsymbol{r} \Psi_{\alpha}^{*}\left(\boldsymbol{r}-\boldsymbol{R}_{\alpha}\right) \hat{O} \Psi_{\alpha}\left(\boldsymbol{r}-\boldsymbol{R}_{\alpha}\right),
$$

where the integral is over the $\alpha^{\text {th }}$-muffin-tin volume and $\hat{O}$ can be replaced by any operator described in Sec. II B.

\section{APPENDIX B: PROPERTIES OF THE ORBITAL RESPONSES}

In this Appendix, we provide detailed calculated results for the orbital susceptibilities. We show in Fig. 12 the calculated dependence of the layer-resolved $\chi^{L}$ tensor elements on the number of Pt monolayers $n$, similar to the results for the spin counterpart shown in Fig. 3. In Figs. 13 and 14 we provide the selfconsistently calculated dependence of the tensor elements on the magnetization angles $\phi$ and $\theta$, respectively, for the $12 \mathrm{Pt} / 2 \mathrm{Co}$ system. The angular dependencies are similar to the ones computed for the induced spin susceptibilities, shown in Figs. 4 and 5, and are even or odd-in- $\boldsymbol{M}$, consistent with the symmetry classification. The slight asymmetry of some tensor elements, e.g., $\chi_{x x}^{L}$ and $\chi_{y y}^{L}$ in Fig. 13, can be ascribed to an angular dependence of the form $\sin 2 \phi /\left(a \cos ^{2} \phi+b \sin ^{2} \phi\right)$, with nonequal constants $a$ and $b$.

In Fig. 15, lastly, we provide the computed magnetizationdirection dependence of the $\chi^{L}$ for magnetization directions $\boldsymbol{M} \| \boldsymbol{u}_{z}$ to $\boldsymbol{M} \| \boldsymbol{u}_{x}$. The similar shape of the curves and the very similar values of the $\chi^{L}$ tensor elements illustrates the mapping according to the employed classification.
[1] J. C. Slonczewski, Current-driven excitation of magnetic multilayers, J. Magn. Magn. Mater. 159, L1 (1996).

[2] L. Berger, Emission of spin waves by a magnetic multilayer traversed by a current, Phys. Rev. B 54, 9353 (1996).

[3] M. Tsoi, A. G. M. Jansen, J. Bass, W. C. Chiang, M. Seck, V. Tsoi, and P. Wyder, Excitation of a Magnetic Multilayer by an Electric Current, Phys. Rev. Lett. 80, 4281 (1998).

[4] E. B. Myers, D. C. Ralph, J. A. Katine, R. N. Louie, and R. A. Buhrman, Current-induced switching of domains in magnetic multilayer devices, Science 285, 867 (1999).
[5] J. E. Wegrowe, D. Kelly, Y. Jaccard, Ph. Guittienne, and J. Ph. Ansermet, Current-induced magnetization reversal in magnetic nanowires, Europhys. Lett. 45, 626 (1999).

[6] J. A. Katine, F. J. Albert, R. A. Buhrman, E. B. Myers, and D. C. Ralph, Current-Driven Magnetization Reversal and SpinWave Excitations in $\mathrm{Co} / \mathrm{Cu} / \mathrm{Co}$ Pillars, Phys. Rev. Lett. 84, 3149 (2000).

[7] A. Brataas, A. D. Kent, and H. Ohno, Current-induced torques in magnetic materials, Nat. Mater. 11, 372 (2012).

[8] J. A. Katine and E. E. Fullerton, Device implications of spintransfer torques, J. Magn. Magn. Mater. 320, 1217 (2008). 
[9] K. Jabeur, G. Di Pendina, and G. Prenat, Study of spin transfer torque (STT) and spin orbit torque (SOT) magnetic tunnel junctions (MTJS) at advanced CMOS technology nodes, Electr. Electron. Engineer. Inter. J (ELELIJ) 6, 01 (2017).

[10] M. I. Miron, G. Gaudin, S. Auffret, B. Rodmacq, A. Schuhl, S. Pizzini, J. Vogel, and P. Gambardella, Current-driven spin torque induced by the Rashba effect in a ferromagnetic metal layer, Nat. Mater. 9, 230 (2010)

[11] M. I. Miron, K. Garello, G. Gaudin, P. J. Zermatten, M. V. Costache, S. Auffret, S. Bandiera, B. Rodmacq, A. Schuhl, and P. Gambardella, Perpendicular switching of a single ferromagnetic layer induced by in-plane current injection, Nature (London) 476, 189 (2011).

[12] L. Liu, T. Moriyama, D. C. Ralph, and R. A. Buhrman, SpinTorque Ferromagnetic Resonance Induced by the Spin Hall Effect, Phys. Rev. Lett. 106, 036601 (2011).

[13] L. Liu, C. F. Pai, Y. Li, H. W. Tseng, D. C. Ralph, and R. A. Buhrman, Spin-torque switching with the giant spin Hall effect of tantalum, Science 336, 555 (2012).

[14] J. Kim, J. Sinha, M. Hayashi, M. Yamanouchi, S. Fukami, T. Suzuki, S. Mitani, and H. Ohno, Layer thickness dependence of the current-induced effective field vector in $\mathrm{Ta}|\mathrm{CoFeB}| \mathrm{MgO}$, Nat. Mater. 12, 240 (2013).

[15] X. Fan, J. Wu, Y. Chen, M. J. Jerry, H. Zhang, and J. Q. Xiao, Observation of the nonlocal spin-orbital effective field, Nat. Commun. 4, 1799 (2013).

[16] C. K. Safeer, E. Jué, A. Lopez, L. Buda-Prejbeanu, S. Auffret, S. Pizzini, O. Boulle, I. M. Miron, and G. Gaudin, Spin-orbit torque magnetization switching controlled by geometry, Nat. Nanotechnol. 11, 143 (2016).

[17] M. Baumgartner, K. Garello, J. Mendil, C. O. Avci, E. Grimaldi, C. Murer, J. Feng, M. Gabureac, C. Stamm, Y. Acremann, S. Finizio, S. Wintz, J. Raabe, and P. Gambardella, Spatially and time-resolved magnetization dynamics driven by spin-orbit torques, Nat. Nanotechnol. 12, 980 (2017).

[18] M. I. D'yakonov and V. I. Perel', Possibility of orienting electron spins with current, JETP Lett. 13, 467 (1971).

[19] J. E. Hirsch, Spin Hall Effect, Phys. Rev. Lett. 83, 1834 (1999).

[20] V. M. Edelstein, Spin polarization of conduction electrons induced by electric current in two-dimensional asymmetric electron systems, Solid State Commun. 73, 233 (1990).

[21] Y. K. Kato, R. C. Myers, A. C. Gossard, and D. D. Awschalom, Current-Induced Spin Polarization in Strained Semiconductors, Phys. Rev. Lett. 93, 176601 (2004).

[22] J. Wunderlich, B. Kaestner, J. Sinova, and T. Jungwirth, Experimental Observation of the Spin-Hall Effect in a TwoDimensional Spin-Orbit Coupled Semiconductor System, Phys. Rev. Lett. 94, 047204 (2005).

[23] A. Hoffmann, Spin Hall effects in metals, IEEE Trans. Magn. 49, 5172 (2013).

[24] J. Sinova, S. O. Valenzuela, J. Wunderlich, C. H. Back, and T. Jungwirth, Spin Hall effects, Rev. Mod. Phys. 87, 1213 (2015).

[25] Y. A. Bychkov and E. I. Rashba, Properties of a 2D electron gas with lifted spectral degeneracy, JETP Lett. 39, 78 (1984).

[26] C. Ciccarelli, L. Anderson, V. Tshitoyan, A. J. Ferguson, F. Gerhard, C. Gould, L. W. Molenkamp, J. Gayles, J. Železný, L. Šmejkal, Z. Yuan, J. Sinova, F. Freimuth, and T. Jungwirth, Room-temperature spin-orbit torque in NiMnSb, Nat. Phys. 12, 855 (2016).
[27] F. Freimuth, S. Blügel, and Y. Mokrousov, Spin-orbit torques in $\mathrm{Co} / \mathrm{Pt}(111)$ and $\mathrm{Mn} / \mathrm{W}(001)$ magnetic bilayers from first principles, Phys. Rev. B 90, 174423 (2014).

[28] V. P. Amin and M. D. Stiles, Spin transport at interfaces with spin-orbit coupling: Phenomenology, Phys. Rev. B 94, 104420 (2016).

[29] S. Wimmer, K. Chadova, M. Seemann, D. Ködderitzsch, and H. Ebert, Fully relativistic description of spin-orbit torques by means of linear response theory, Phys. Rev. B 94, 054415 (2016).

[30] F. Mahfouzi and N. Kioussis, First-principles study of the angular dependence of the spin-orbit torque in $\mathrm{Pt} / \mathrm{Co}$ and $\mathrm{Pd} / \mathrm{Co}$ bilayers, Phys. Rev. B 97, 224426 (2018).

[31] A. J. Berger, E. R. J. Edwards, H. T. Nembach, A. D. Karenowska, M. Weiler, and T. J. Silva, Inductive detection of fieldlike and dampinglike ac inverse spin-orbit torques in ferromagnet/normal-metal bilayers, Phys. Rev. B 97, 094407 (2018).

[32] K. D. Belashchenko, A. A. Kovalev, and M. van Schilfgaarde, First-principles calculation of spin-orbit torque in a $\mathrm{Co} / \mathrm{Pt}$ bilayer, Phys. Rev. Mater. 3, 011401(R) (2019).

[33] F. Mahfouzi, R. Mishra, P.-H. Chang, H. Yang, and N. Kioussis, Microscopic origin of spin-orbit torque in ferromagnetic heterostructures: A first-principles approach, Phys. Rev. B 101, 060405(R) (2020).

[34] X. Fan, H. Celik, J. Wu, C. Ni, K.-J. Lee, V. O. Lorenz, and J. Q. Xiao, Quantifying interface and bulk contributions to spin-orbit torque in magnetic bilayers, Nat. Commun. 5, 3042 (2014).

[35] Y. Du, H. Gamou, S. Takahashi, S. Karube, M. Kohda, and J. Nitta, Disentanglement of Spin-Orbit Torques in Pt/Co Bilayers with the Presence of Spin Hall Effect and Rashba-Edelstein Effect, Phys. Rev. Applied 13, 054014 (2020).

[36] L. Zhu and R. A. Buhrman, Absence of Significant SpinCurrent Generation in Ti/Fe-Co-B Bilayers with Strong Interfacial Spin-Orbit Coupling, Phys. Rev. Applied 15, L031001 (2021).

[37] A. Manchon, J. Železný, I. M. Miron, T. Jungwirth, J. Sinova, A. Thiaville, K. Garello, and P. Gambardella, Current-induced spin-orbit torques in ferromagnetic and antiferromagnetic systems, Rev. Mod. Phys. 91, 035004 (2019).

[38] I. V. Tokatly, E. E. Krasovskii, and G. Vignale, Current-induced spin polarization at the surface of metallic films: A theorem and an ab initio calculation, Phys. Rev. B 91, 035403 (2015).

[39] L. Wang, R. J. H. Wesselink, Y. Liu, Z. Yuan, K. Xia, and P. J. Kelly, Giant Room Temperature Interface Spin Hall and Inverse Spin Hall Effects, Phys. Rev. Lett. 116, 196602 (2016).

[40] V. P. Amin, J. Zemen, and M. D. Stiles, Interface-Generated Spin Currents, Phys. Rev. Lett. 121, 136805 (2018).

[41] G. Y. Guo, Y. Yao, and Q. Niu, Ab Initio Calculation of the Intrinsic Spin Hall Effect in Semiconductors, Phys. Rev. Lett. 94, 226601 (2005).

[42] T. Tanaka, H. Kontani, M. Naito, T. Naito, D. S. Hirashima, K. Yamada, and J. Inoue, Intrinsic spin Hall effect and orbital Hall effect in $4 d$ and $5 d$ transition metals, Phys. Rev. B 77, 165117 (2008).

[43] H. Kontani, T. Tanaka, D. S. Hirashima, K. Yamada, and J. Inoue, Giant Orbital Hall Effect in Transition Metals: Origin of Large Spin and Anomalous Hall Effects, Phys. Rev. Lett. 102, 016601 (2009). 
[44] D. Go, D. Jo, C. Kim, and H.-W. Lee, Intrinsic Spin and Orbital Hall Effects from Orbital Texture, Phys. Rev. Lett. 121, 086602 (2018).

[45] D. Go and H.-W. Lee, Orbital torque: Torque generation by orbital current injection, Phys. Rev. Research 2, 013177 (2020).

[46] L. Salemi, M. Berritta, A. K. Nandy, and P. M. Oppeneer, Orbitally dominated Rashba-Edelstein effect in noncentrosymmetric antiferromagnets, Nat. Commun. 10, 5381 (2019).

[47] C. Stamm, C. Murer, Y. Acremann, M. Baumgartner, R. Gort, S. Däster, A. Kleibert, K. Garello, J. Feng, M. Gabureac, Z. Chen, J. Stöhr, and P. Gambardella, X-ray spectroscopy of current-induced spin-orbit torques and spin accumulation in $\mathrm{Pt} / 3 d$-transition-metal bilayers, Phys. Rev. B 100, 024426 (2019).

[48] J. Xiao, Y. Liu, and B. Yan, Detection of the orbital Hall effect by the orbital-spin conversion, arXiv:2010.01970 [condmat.mtrl-sci].

[49] P. Blaha, K. Schwarz, G. K. H. Madsen, D. Kvasnicka, J. Luitz, R. Laskowski, F. Tran, and L. D. Marks, WIEN2K, An Augmented Plane Wave + Local Orbitals Program for Calculating Crystal Properties (Karlheinz Schwarz, Techn. Universität Wien, Austria, 2018).

[50] G. Y. Guo, S. Murakami, T.-W. Chen, and N. Nagaosa, Intrinsic Spin Hall Effect in Platinum: First-Principles Calculations, Phys. Rev. Lett. 100, 096401 (2008).

[51] C. Stamm, C. Murer, M. Berritta, J. Feng, M. Gabureac, P. M. Oppeneer, and P. Gambardella, Magneto-Optical Detection of the Spin Hall Effect in Pt and W Thin Films, Phys. Rev. Lett. 119, 087203 (2017).

[52] J. M. Soler, E. Artacho, J. D. Gale, A. García, J. Junquera, P. Ordejón, and D. Sánchez-Portal, The SIESTA method for ab initio order-N materials simulation, J. Phys.: Condens. Matter 14, 2745 (2002).

[53] J. Železný, H. Gao, A. Manchon, F. Freimuth, Y. Mokrousov, J. Zemen, J. Mašek, J. Sinova, and T. Jungwirth, Spin-orbit torques in locally and globally noncentrosymmetric crystals: Antiferromagnets and ferromagnets, Phys. Rev. B 95, 014403 (2017).

[54] S. Zhang, Spin Hall Effect in the Presence of Spin Diffusion, Phys. Rev. Lett. 85, 393 (2000).

[55] H. Kurt, R. Loloee, K. Eid, W. P. Pratt, and J. Bass, Spinmemory loss at $4.2 \mathrm{~K}$ in sputtered $\mathrm{Pd}$ and $\mathrm{Pt}$ and at $\mathrm{Pd} / \mathrm{Cu}$ and $\mathrm{Pt} / \mathrm{Cu}$ interfaces, Appl. Phys. Lett. 81, 4787 (2003).

[56] J. C. Rojas-Sánchez, N. Reyren, P. Laczkowski, W. Savero, J. P. Attané, C. Deranlot, M. Jamet, J. M. George, L. Vila, and H. Jaffrès, Spin Pumping and Inverse Spin Hall Effect in Platinum: The Essential Role of Spin-Memory Loss at Metallic Interfaces, Phys. Rev. Lett. 112, 106602 (2014).

[57] H. Y. T. Nguyen, W. P. Pratt, and J. Bass, Spin-flipping in Pt and at Co/Pt interfaces, J. Magn. Magn. Mater. 361, 30 (2014).

[58] K. Dolui and B. K. Nikolić, Spin-memory loss due to spin-orbit coupling at ferromagnet/heavy-metal interfaces: Ab initio spindensity matrix approach, Phys. Rev. B 96, 220403(R) (2017).

[59] X. Tao, Q. Liu, B. Miao, R. Yu, Z. Feng, L. Sun, B. You, J. Du, K. Chen, S. Zhang, L. Zhang, Z. Yuan, D. Wu, and H. Ding, Self-consistent determination of spin Hall angle and spin diffusion length in Pt and Pd: The role of the interface spin loss, Sci. Adv. 4, aat1670 (2018).
[60] M. Kimata, H. Chen, K. Kondou, S. Sugimoto, P. K. Muduli, M. Ikhlas, Y. Omori, T. Tomita, A. H. MacDonald, S. Nakatsuji, and Y. Otani, Magnetic and magnetic-inverse spin Hall effects in a non-collinear antiferromagnet, Nature (London) 565, 627 (2019).

[61] T. C. Chuang, D. Qu, S. Y. Huang, and S. F. Lee, Magnetizationdependent spin Hall effect in a perpendicular magnetized film, Phys. Rev. Research 2, 032053(R) (2020).

[62] A. M. Humphries, T. Wang, E. R. J. Edwards, S. R. Allen, J. M. Shaw, H. T. Nembach, J. Q. Xiao, T. J. Silva, and X. Fan, Observation of spin-orbit effects with spin rotation symmetry, Nat. Commun. 8, 911 (2017).

[63] T. Wang, S. Lendinez, M. B. Jungfleisch, J. Kolodzey, J. Q. Xiao, and X. Fan, Detection of spin-orbit torque with spin rotation symmetry, Appl. Phys. Lett. 116, 012404 (2020).

[64] G. Qu, K. Nakamura, and M. Hayashi, Magnetization direction dependent spin Hall effect in $3 d$ ferromagnets, Phys. Rev. B 102, 144440 (2020).

[65] C. Safranski, J. Z. Sun, J.-W. Xu, and A. D. Kent, Planar Hall Driven Torque in a Ferromagnet/Nonmagnet/Ferromagnet System, Phys. Rev. Lett. 124, 197204 (2020).

[66] M.-H. Nguyen, D. C. Ralph, and R. A. Buhrman, Spin Torque Study of the Spin Hall Conductivity and Spin Diffusion Length in Platinum Thin Films with Varying Resistivity, Phys. Rev. Lett. 116, 126601 (2016).

[67] C. O. Avci, K. Garello, M. Gabureac, A. Ghosh, A. Fuhrer, S. F. Alvarado, and P. Gambardella, Interplay of spin-orbit torque and thermoelectric effects in ferromagnet/normal-metal bilayers, Phys. Rev. B 90, 224427 (2014).

[68] A. Johansson, J. Henk, and I. Mertig, Edelstein effect in Weyl semimetals, Phys. Rev. B 97, 085417 (2018).

[69] P. M. Haney, H.-W. Lee, K.-J. Lee, A. Manchon, and M. D. Stiles, Current induced torques and interfacial spin-orbit coupling: Semiclassical modeling, Phys. Rev. B 87, 174411 (2013).

[70] D. Jo, D. Go, and H.-W. Lee, Gigantic intrinsic orbital Hall effects in weakly spin-orbit coupled metals, Phys. Rev. B 98, 214405 (2018).

[71] J. Kim, D. Go, H. Tsai, D. Jo, K. Kondou, H.-W. Lee, and Y. Otani, Nontrivial torque generation by orbital angular momentum injection in ferromagnetic-metal/ $\mathrm{Cu} / \mathrm{Al}_{2} \mathrm{O}_{3}$ trilayers, Phys. Rev. B 103, L020407 (2021).

[72] S. Ding, A. Ross, D. Go, L. Baldrati, Z. Ren, F. Freimuth, S. Becker, F. Kammerbauer, J. Yang, G. Jakob, Y. Mokrousov, and M. Kläui, Harnessing Orbital-to-Spin Conversion of Interfacial Orbital Currents for Efficient Spin-Orbit Torques, Phys. Rev. Lett. 125, 177201 (2020).

[73] Y. Tazaki, Y. Kageyama, H. Hayashi, T. Harumoto, T. Gao, J. Shi, and K. Ando, Current-induced torque originating from orbital current, arXiv:2004.09165 [cond-mat.mtrl-sci].

[74] D. Go, D. Jo, T. Gao, K. Ando, S. Blügel, H.-W. Lee, and Y. Mokrousov, Orbital Rashba effect in a surface-oxidized $\mathrm{Cu}$ film, Phys. Rev. B 103, L121113 (2021)

[75] F. Freimuth, S. Blügel, and Y. Mokrousov, Direct and inverse spin-orbit torques, Phys. Rev. B 92, 064415 (2015).

[76] D. Go, F. Freimuth, J.-Ph. Hanke, F. Xue, O. Gomonay, K.-J. Lee, S. Blügel, P. M. Haney, H.-W. Lee, and Y. Mokrousov, Theory of current-induced angular momentum transfer dynamics in spin-orbit coupled systems, Phys. Rev. Research 2, 033401 (2020). 
[77] X. Chen, Y. Liu, G. Yang, H. Shi, C. Hu, M. Li, and H. Zeng, Giant antidamping orbital torque originating from the orbital Rashba-Edelstein effect in ferromagnetic heterostructures, Nat. Commun. 9, 2569 (2018).

[78] S. Ding, Z. Liang, D. Go, C. Yun, M. Xue, Z. Liu, S. Becker, W. Yang, H. Du, C. Wang, Y. Yang, G. Jakob, M. Kläui, Y. Mokrousov, and J. Yang, Observation of the orbital Rashba-Edelstein magnetoresistance, arXiv:2105.04495 [condmat.mtrl-sci].

[79] U. Nowak, Classical spin models, in Handbook of Magnetism and Advanced Magnetic Materials, edited by $\mathrm{H}$. Kronmüller and S. Parkin (J. Wiley Sons, Chichester, 2007), Vol. 2.

[80] R. F. L. Evans, W. J. Fan, P. Chureemart, T. A. Ostler, M. O. A. Ellis, and R. W. Chantrell, Atomistic spin model simulations of magnetic nanomaterials, J. Phys.: Condens. Matter 26, 103202 (2014).

[81] M. B. Jungfleisch, W. Zhang, R. Winkler, and A. Hoffmann, Spin-orbit torques and spin dynamics, in Spin Physics in Semi- conductors, edited by M. Dyakonov (Springer, Berlin, 2017), Vol. 157, pp. 355-385.

[82] K. T. Yamada, Y. Shiota, T. Koyama, Y. Kotani, K. Toyoki, H. Mizuno, F. Ando, K. Nakamura, T. Nakamura, D. Chiba, and T. Ono, Orbital-dependent electric field effect on magnetism in ultrathin cobalt, Phys. Rev. B 102, 100407(R) (2020).

[83] A. Obinata, T. Koyama, F. Matsukura, K. Nakamura, and D. Chiba, Electric-field effect on magnetic moments in Co ultrathin films deposited on Pt, Appl. Phys. Lett. 118, 152405 (2021).

[84] H. J. Monkhorst and J. D. Pack, Special points for Brillouinzone integrations, Phys. Rev. B 13, 5188 (1976).

[85] J. P. Perdew, A. Ruzsinszky, G. I. Csonka, O. A. Vydrov, G. E. Scuseria, L. A. Constantin, X. Zhou, and K. Burke, Restoring the Density-Gradient Expansion for Exchange in Solids and Surfaces, Phys. Rev. Lett. 100, 136406 (2008).

[86] J. Kuneš, P. Novák, M. Diviš, and P. M. Oppeneer, Magnetic, magneto-optical, and structural properties of URhAl from firstprinciples calculations, Phys. Rev. B 63, 205111 (2001). 